\title{
Targeting tumor-associated macrophages and granulocytic myeloid-derived suppressor cells augments PD-1 blockade in cholangiocarcinoma
}

\author{
Emilien Loeuillard, ${ }^{1}$ Jingchun Yang, ${ }^{1}$ EeeLN Buckarma, ${ }^{2}$ Juan Wang, ${ }^{1}$ Yuanhang Liu, ${ }^{3}$ Caitlin Conboy, ${ }^{4}$ Kevin D. Pavelko, ${ }^{5}$ Ying Li, ${ }^{3}$ \\ Daniel O'Brien, ${ }^{3}$ Chen Wang, ${ }^{3}$ Rondell P. Graham, ${ }^{6}$ Rory L. Smoot, ${ }^{2}$ Haidong Dong, ${ }^{5,7}$ and Sumera Ilyas ${ }^{1}$
}

Division of Gastroenterology and Hepatology, ${ }^{2}$ Department of Surgery, ${ }^{3}$ Department of Health Sciences Research, ${ }^{4}$ Division of Medical Oncology, ${ }^{5}$ Department of Immunology, ${ }^{6}$ Department of Laboratory Medicine and Pathology, and Department of Urology, Mayo Clinic College of Medicine and Science, Rochester, Minnesota, USA.

\begin{abstract}
Immune checkpoint blockade (ICB) has revolutionized cancer therapeutics. Desmoplastic malignancies, such as cholangiocarcinoma (CCA), have an abundant tumor immune microenvironment (TIME). However, to date, ICB monotherapy in such malignancies has been ineffective. Herein, we identify tumor-associated macrophages (TAMs) as the primary source of programmed death-ligand 1 (PD-L1) in human and murine CCA. In a murine model of CCA, recruited PD-L1+ TAMs facilitated CCA progression. However, TAM blockade failed to decrease tumor progression due to a compensatory emergence of granulocytic myeloid-derived suppressor cells (G-MDSCs) that mediated immune escape by impairing T cell response. Singlecell RNA sequencing (scRNA-Seq) of murine tumor G-MDSCs highlighted a unique ApoE G-MDSC subset enriched with TAM blockade; further analysis of a human scRNA-Seq data set demonstrated the presence of a similar G-MDSC subset in human CCA. Finally, dual inhibition of TAMs and G-MDSCs potentiated ICB. In summary, our findings highlight the therapeutic potential of coupling ICB with immunotherapies targeting immunosuppressive myeloid cells in CCA.
\end{abstract}

\section{Introduction}

Cholangiocarcinoma (CCA) is the most common biliary malignancy and the second most common primary hepatic malignancy (1). CCAs are highly lethal neoplasms with limited therapeutic options and a dismal 5-year survival rate of less than $10 \%$ (2, 3 ). The majority of patients present with advanced disease not amenable to potentially curative options, such as surgical resection or neoadjuvant chemoradiation followed by liver transplantation (1). The available systemic therapies for patients with advanced disease are of limited efficacy, with an overall survival rate of less than 1 year in patients treated with gemcitabine and cisplatin, the current standard of care (4). Hence, there is a critical need for the development of highly effective medical therapies for patients with advanced stage CCA.

The advent of immune-directed therapies has revolutionized the treatment of human cancers. Induction of immune checkpoints, such as programmed death-1 (PD-1) and its ligand, PD-L1, mediates tumor immune evasion $(5,6)$. Immune checkpoint blockade (ICB) employs antibody-based therapies targeting these checkpoints in an effort to unleash preexisting adaptive immunity. Desmoplastic malignancies such as CCA have an abundant tumor immune microenvironment (TIME). However, to date, ICB monotherapy in such malignancies has been disap-

Conflict of interest: The authors have declared that no conflict of interest exists. Copyright: (c) 2020, American Society for Clinical Investigation.

Submitted: February 7, 2020; Accepted: July 2, 2020; Published: September 14, 2020. Reference information: / Clin Invest. 2020;130(10):5380-5396.

https://doi.org/10.1172/JCI137110. pointing, with a response rate of less than $10 \%$ (7), implying that these cancers have a poorly immunogenic or "cold" TIME and require further strategies for effective immunotherapy (8). The suboptimal response to ICB monotherapy may be due to immunosuppressive mechanisms involving the innate immune system. Indeed, the TIME of highly lethal, difficult-to-treat, desmoplastic malignancies, such as CCA or pancreatic ductal adenocarcinoma, has an abundance of immunosuppressive myeloid cells, including tumor-associated macrophages (TAMs) and myeloid-derived suppressor cells (MDSCs) $(9,10)$. Macrophage differentiation toward a protumor phenotype is driven, in part, by a paracrine signaling loop involving tumor CSF1 $(11,12)$. Consequently, inhibition of the CSF1 receptor (CSF1R) has been used as an approach to depleting protumor macrophages in preclinical models of cancer (13-15). MDSCs are immature myeloid cells that have morphologic and phenotypic features of neutrophils and monocytes (16). TAMs and MDSCs facilitate tumor progression via suppression of cytotoxic $\mathrm{T}$ lymphocytes (CTLs), enhanced angiogenesis, tumor invasion, and metastasis $(16,17)$.

Expression of PD-L1 is an important mechanism implicated in tumor immune escape (18). Although the role of PD-L1 in tumor biology is under intense investigation, the majority of the existing body of work has focused on PD-L1 expression on cancer cells and its role in suppression of CTL function. However, PD-L1 can also be expressed by TAMs, and in some cancers, PD-L1 expression by host myeloid cells is more effective than cancer cell expression of PD-L1 in suppressing CTL function (19-21). Moreover, MDSCs may also suppress CTL activity by PD-L1-dependent and -independent mechanisms (22). 
A

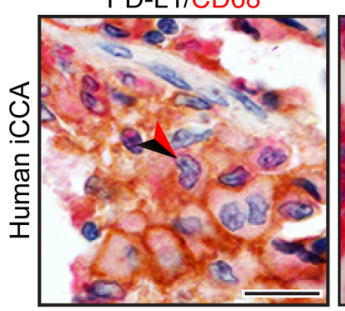

PD-L1/CK19

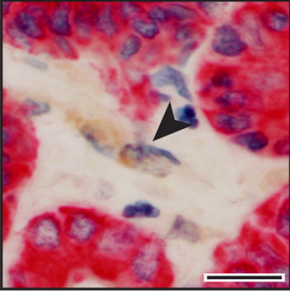

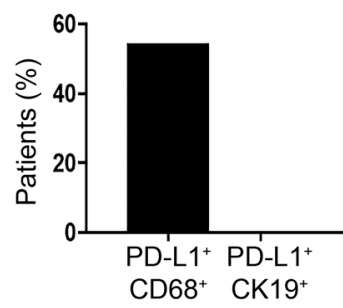

B

Human CCA

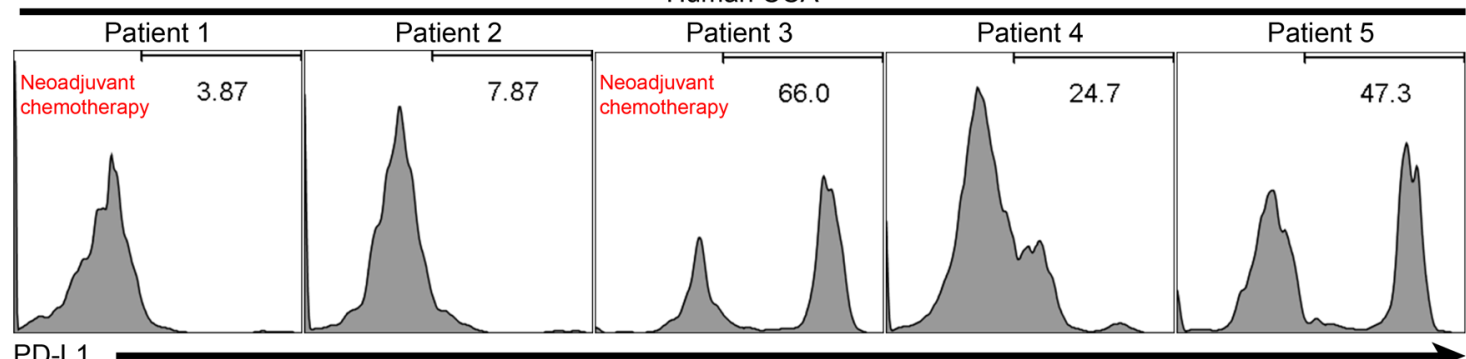

Gate on $\mathrm{CD} 68^{+} \mathrm{CD} 11 \mathrm{~b}^{+}$macrophages

C

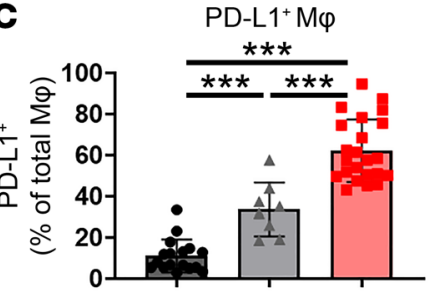

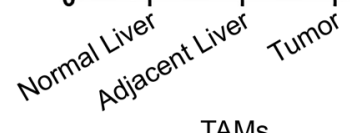

D
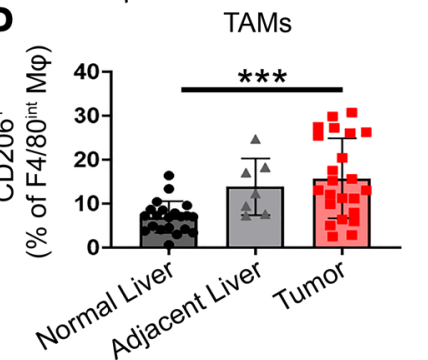

E

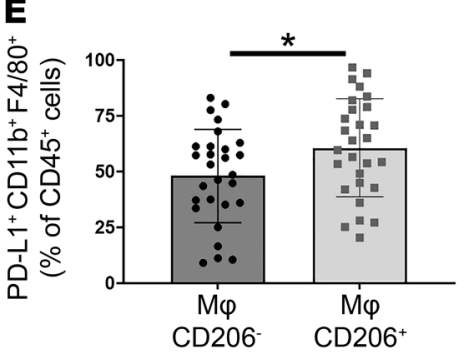

PD-L1

Gate on $\mathrm{CD} 11 \mathrm{~b}^{+} \mathrm{F} 4 / 80^{+}$macrophages

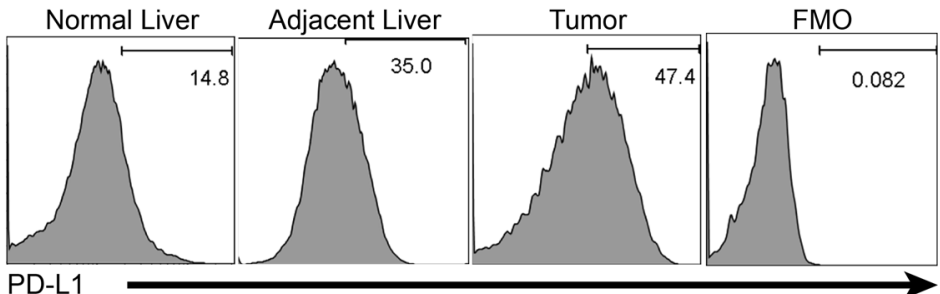

C
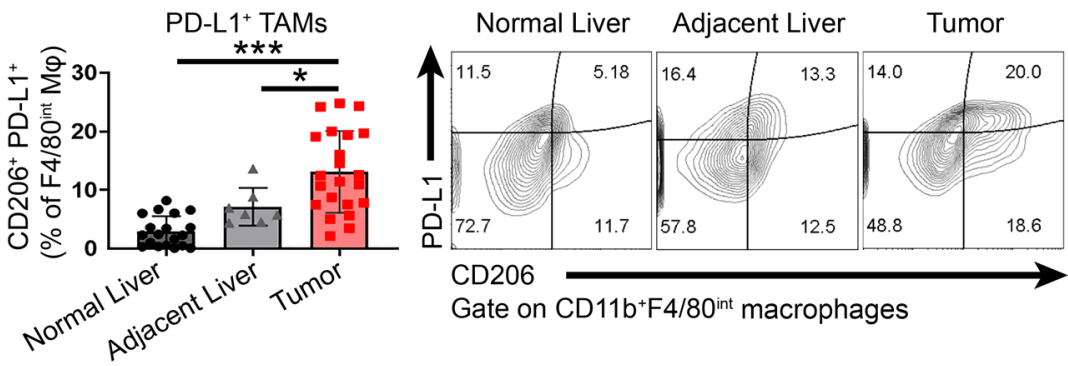

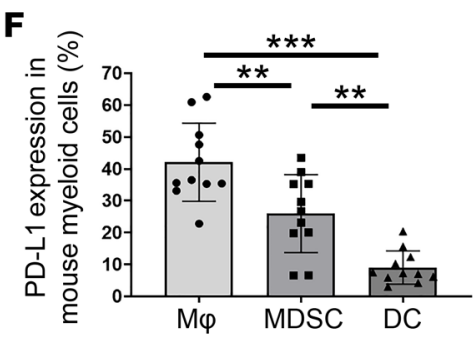

Figure 1. TAMs are the predominant source of PD-L1 in CCA. (A) Representative images (left and middle panels) of PD-L1 (brown staining, black arrowhead) plus CD68 (red staining, red arrowhead) coimmunostaining ( $n=33$ ) and PD-L1 (brown staining) plus CK-19 (red staining) coimmunostaining ( $n=18$ ) in human resected CCA specimens. Percentage of patients with positive PD-L1/CD68 costaining and PD-L1/CK19 costaining, respectively (right panel). Scale bars: $40 \mu \mathrm{m}$. (B) Histograms show expression of PD-L1+ macrophages in human CCA tumors. (C-F) Flow cytometry analysis of normal WT mouse livers (from WT mice without tumors) as well as adjacent livers and tumors of mice 28 days after orthotopic implantation of $1 \times 10^{6} \mathrm{SB}$ (murine CCA) cells. (C) Percentage of PD-L1 ${ }^{+}$macrophages (M $\varphi$ ) of total

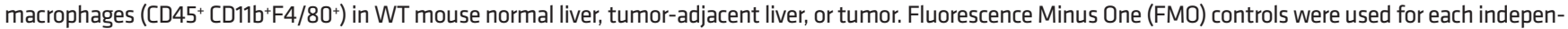
dent experiment to establish gates (See Supplemental Figure 1A for gating strategy) ( $n \geq 8$ ). Representative histograms show expression of PD-L1+ macrophages. (D) Percentage of CD206+ TAMs (left panel) and PD-L1+CD206+ TAMs (middle panel) of F4/80 int macrophages (CD45 ${ }^{+}$CD11 $\mathrm{b}^{+} \mathrm{F} 4 / 8 \mathrm{O}^{\text {int }}$ ) in WT mouse liver, tumor-adjacent liver, or tumor. Representative contour plots (right panel) show CD206 and PD-L1 expression of F4/80 int macrophages $(n \geq 7)$. (E) Percentage of PD-L1+CD206- macrophages or PD-L1+CD206+ macrophages (CD11 b'F4/80+) of CD45+ cells from SB tumors $(n=28)$. (F) Percentage of PD-L1 expression in myeloid cells from SB tumors. Macrophages, CD45+PD-L1+CD11b+F4/80+; MDSCs, CD45+PD-L1+CD11c-CD11b+F4/80-CR-1+; DCs, CD45+PD-L1+CD11c chi; $(n=11)$. Data are represented as mean \pm SD. Unpaired Student's $t$ test (E) and 1-way ANOVA with Bonferroni's post hoc test $(\mathbf{C}, \mathbf{D}$, and $\mathbf{F})$ were used. ${ }^{*} P<0.05 ;{ }^{* *} P<0.01 ;{ }^{* *} P<0.001$. 
We have developed an oncogene-driven murine model of CCA (23). In this genetic model, the biliary tree of C57BL/6 mice is directly transduced with constitutively active human yes associated protein (YAP) along with murine myristoylated Akt as a permissive factor. Phenotypic characterization of these tumors harvested 10 weeks after biliary instillation demonstrated that they recapitulate the human disease. RNA-Seq analysis identified substantive overlap in gene expression of the mouse tumors with human CCA. Malignant murine cell lines derived from this model can be implanted in an orthotopic fashion into livers of immunocompetent mice (C57BL/6 background), generating a syngeneic mouse model, which we will simply define as having SB tumors (24). The mice develop large tumors with typically one dominant nodule that can be easily separated from the adjacent liver. This enables study of the immune microenvironment of the tumor as well as the adjacent liver and thus is an ideal model for studying the immunobiology of this desmoplastic malignancy.

Herein, we employ our orthotopic murine CCA model to uncover the interplay between immunosuppressive myeloid cells and their response to immunotherapies. We demonstrate that PD-L1+ TAMs are abundant in human CCA as well as in our murine model of CCA. However, TAM blockade failed to reduce CCA tumor burden. This observation is likely due to compensatory accumulation of immunosuppressive granulocytic MDSCs (G-MDSCs). Dual inhibition of TAMs and G-MDSCs was necessary to potentiate ICB in our murine CCA model. These data suggest that combination immune therapies targeting macrophages and MDSCs are a promising therapeutic approach for desmoplastic cancers.

\section{Results}

TAMs are the predominant source of PD-L1 in CCA. PD-L1 expression on both the host myeloid cells and tumor cells has been implicated in suppression of the antitumor immune response (20, 21). As macrophages are abundant in desmoplastic malignancies such as CCA, we assessed PD-L1 expression on macrophages and tumor cells in resected human CCA specimens. Using costaining for PD-L1 plus CD68 (a macrophage marker) and PD-L1 plus cytokeratin-19 (CK-19) (a CCA marker), we demonstrated that PD-L1 is expressed predominantly on macrophages rather than cancer cells in human CCA (Figure 1A and Supplemental Figure 1A; supplemental material available online with this article; https:/doi. org/10.1172/JCI137110DS1). Next, we assessed for the presence of $\mathrm{PD}-\mathrm{L}^{+}$macrophages in the tumors of 5 patients undergoing surgical resection; 2 patients had received neoadjuvant chemoradiation with gemcitabine and cisplatin. Flow cytometry was conducted on human CCA tumor immediately following surgical resection. $\mathrm{CD} 11 \mathrm{~b}^{+} \mathrm{CD} 68^{+} \mathrm{PD}-\mathrm{L}^{+}$macrophages were detected in all 5 patients (Figure $1 \mathrm{~B}$ ).

Approximately $60 \%$ of all macrophages $\left(\mathrm{CD} 11 \mathrm{~b}^{+} \mathrm{F} 4 / 80^{+}\right)$in the SB tumors expressed PD-L1 (Figure 1C). Macrophage PD-L1 expression was also increased in the tumor-adjacent liver compared with WT baseline liver (from normal WT mice without tumor) (Figure 1C). Further characterization of intratumoral macrophage phenotypes in these tumors identified $\mathrm{F} 4 / 80^{\text {hi }}$ and $\mathrm{F} 4 / 80^{\text {int }}$ macrophages $\left(\mathrm{CD} 45^{+} \mathrm{CD} 11 \mathrm{~b}^{+} \mathrm{F} 4 / 80^{+}\right)$subsets (Supplemental Figure 1, B-D). As the preponderance of macrophages in the murine CCA tumors were $\mathrm{F} 4 / 80^{\text {int }}$, with $\mathrm{F} 4 / 80^{\text {hi }}$ only making up a minor proportion of the $\mathrm{CD} 45^{+} \mathrm{CD} 11 \mathrm{~b}^{+} \mathrm{F} 4 / 80^{+}$population, we elected to focus on $\mathrm{F} 4 / 80^{\text {int }}$ macrophages for the remainder of our studies. The percentages of $\mathrm{F} 4 / 80^{\text {int }}$ macrophages that expressed CD206, a scavenger receptor expressed by TAMs (25), and those that were PD-L1 ${ }^{+}\left(\mathrm{CD} 206^{+} \mathrm{PD}-\mathrm{L} 1^{+}\right)$was significantly increased in murine CCA tumors compared with normal liver (Figure 1D). Moreover, the majority of the macrophages that expressed PD-L1 were CD206 ${ }^{+}$, indicating that PD-L1 is expressed on TAMs (Figure $1 \mathrm{E})$. MDSCs and DCs $\left(\mathrm{CD} 45^{+} \mathrm{CD} 11 \mathrm{c}^{\text {hi }}\right)$ were also significantly increased in murine CCA tumors compared with normal liver (Supplemental Figure 1, E and F). Although we identified PD-L1 expression on DCs and MDSCs, the preponderance of PD-L1 in SB tumors was expressed on TAMs (Figure 1F). In aggregate, these data indicate that PD-L1 is predominantly expressed on macrophages in CCA.

Host PD-L1 contributes to CCA progression. The relative contribution of PD-L1 expression in the host immune cell and tumor compartments in CCA is unknown. To examine the relative roles of PD-L1 expression on tumor versus myeloid cells, we employed our syngeneic CCA model and implanted murine CCA cells (SB cells) orthotopically into livers of WT mice and $\mathrm{Pd}-l 1^{-/-}$mice. Interestingly, although the SB cells had abundant PD-L1 expression (Figure 2A), implantation of these cells in $\mathrm{Pd}-l 1^{-/-}$mice resulted in significant reduction in the CCA tumor burden compared with that of WT mice, suggesting that tumor-derived PD-L1 was not the driver of immune escape in these tumors (Figure 2, B and C, and Supplemental Figure 2, A and B). Moreover, CD206 ${ }^{+}$and CD206 ${ }^{+} \mathrm{PD}-\mathrm{L} 1^{+}$TAMs were significantly decreased in SB tumors in Pd-l1-deficient hosts (Figure 2D and Supplemental Figure 2C). Although SB tumors in WT hosts displayed suppression of $\mathrm{CD}^{+}$ $\mathrm{T}$ cell infiltration, SB tumors in Pd-l1-deficient hosts manifested enhanced $\mathrm{CD}^{+} \mathrm{T}$ cell infiltration and increased reactive CTLs, as demonstrated by CD11a expression (Figure 2, E and F, and Supplemental Figure 2C). CD11a, an integrin that is upregulated in effector and memory $\mathrm{CD}^{+} \mathrm{T}$ cells, mediates conjugation between CTLs and target cells and can be used to identify and monitor endogenous tumor-reactive CTLs (26). These results suggest that host myeloid cell PD-L1 is requisite for CCA progression.

Soluble nonfunctional PD-L1 is transferred from murine CCA cells to TAMs. Although host immune cells in $\mathrm{Pd}-\mathrm{l1} 1^{-/-}$mice are devoid of PD-L1, we consistently observed the presence of $\mathrm{PD}-\mathrm{L} 1^{+}$TAMs in $\mathrm{Pd}-\mathrm{l1} 1^{-/-}$mouse tumors, implying direct transfer of PD-L1 from the cancer cells to TAMs (Figure 2D). Hence, we postulated that SB cells, which have abundant PD-L1 expression (Figure 2A), transfer PD-L1 to TAMs. Accordingly, we assessed PD-L1 levels on BM-derived macrophages (BMDMs) isolated from $\mathrm{Pd}-l 1^{-/-}$mice and subsequently cocultured with SB cells. Using flow cytometry analysis, we observed a significant increase in PD-L1 levels on $\mathrm{Pd}-\mathrm{l1}^{-/-} \mathrm{BMDM}$ cocultured with SB cells compared with control $\mathrm{Pd}-\mathrm{l1}^{-/-}$BMDMs (Figure $2 \mathrm{G}$ ). We also tested to determine whether direct cell contact was required for accumulation of PD-L1 on BMDMs. Addition of conditioned medium from SB cells to BMDMs isolated from WT C57BL/6 mice significantly increased BMDM PD-L1 protein levels, as assessed by flow cytometry, implying that direct cell contact is not required for PD-L1 accumulation on BMDMs (Figure 2H). As tumor-derived 
A

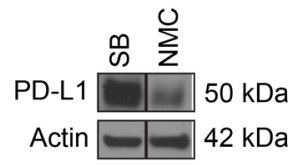

B

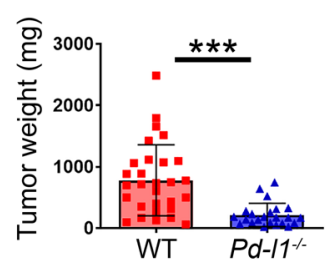

C

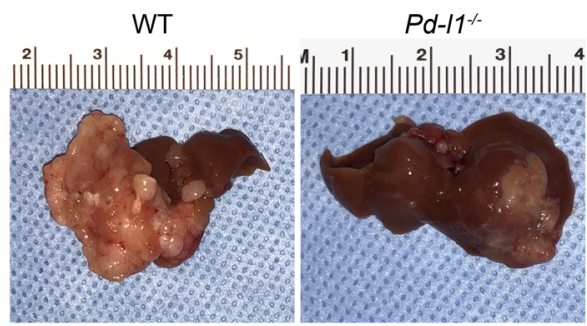

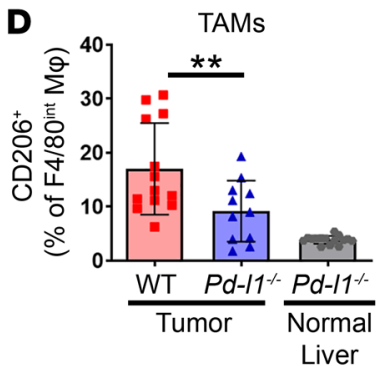

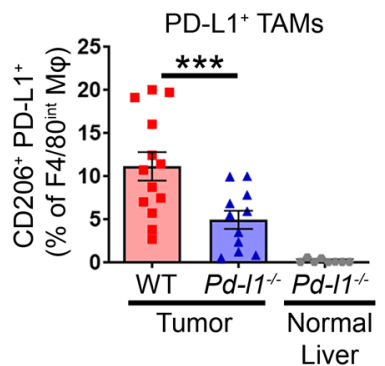

E

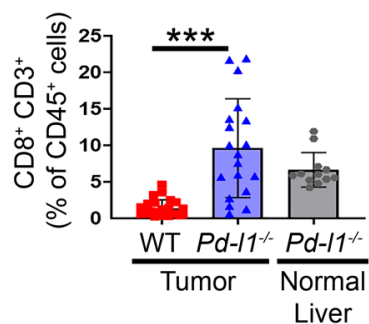

$\mathbf{F}$

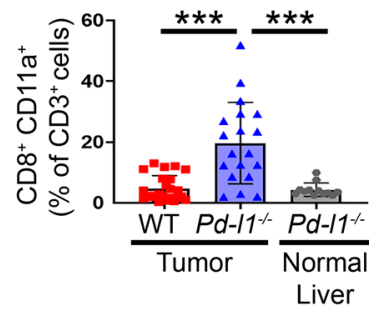

G

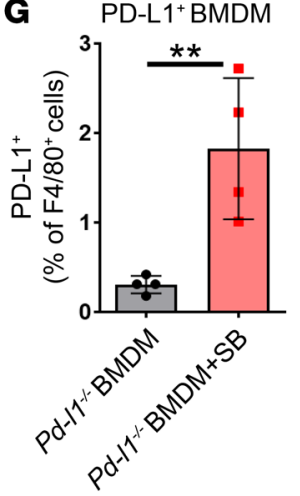

J

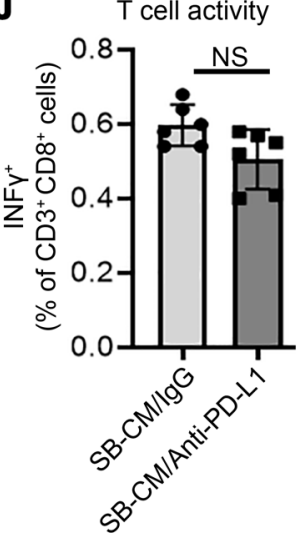

H

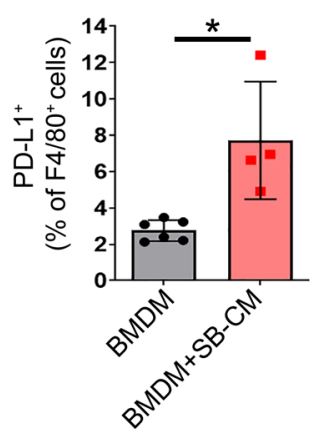

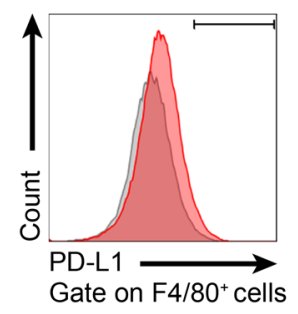

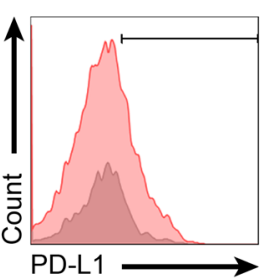

Gate on $\mathrm{F} 4 / 80^{+}$cells

Gate on $\mathrm{F} 4 / 80^{+}$cells

I Soluble PD-L1

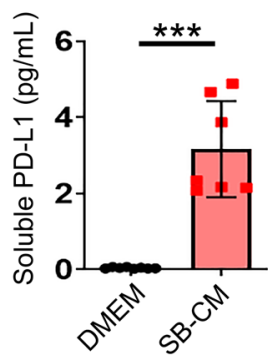

Figure 2. Host PD-L1 contributes to CCA progression. (A) Immunoblot analysis of PD-L1 in mouse CCA cells (SB) and normal mouse cholangiocytes (NMC). (B-F) Tumor growth of 28 days after orthotopic implantation of $1 \times 10^{6} \mathrm{SB}$ cells in WT or Pd-17/-- mouse livers. (B) Average tumor weights in mg of WT or $P d-17^{-1-}$ mice ( $n \geq 23$ ). (C) Representative photographs of livers from B. (D) Percentage of CD206+ TAMs (left panel) and CD206+PD-L1+ TAMs (right panel) of F4/80 int TAMs (CD45 ${ }^{+}$

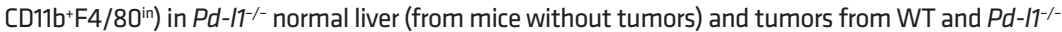
mice $(n \geq 8)$. (E) Percentage of $C D 8^{+} C D 3^{+} T$ CTLs of $C D 45^{+}$cells in $P d-11^{-/-}$normal liver and tumors from WT and $P d-11^{-1-}$ mice $(n \geq 12)$. (F) Percentage of CD8 ${ }^{+} \mathrm{CD} 11 a^{+}$reactive CTLs of CD45+CD3 ${ }^{+}$cells in

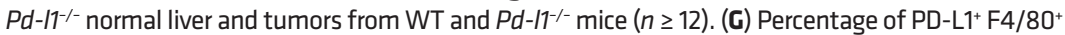
BMDMs after 72 hours of coculture in vitro with $\mathrm{SB}$ cells (ratio 1:1). BMDMs were isolated from WT mice $(n=4)$. (H) Percentage of PD-L1+ $\mathrm{F} 4 / 80^{+}$BMDMs after 24 hours of treatment with conditioned medium (CM) from SB cells $(1 \mathrm{~mL})$. BMDMs were isolated from WT mice $(n=4)$. (I) Concentration $(\mathrm{pg} / \mathrm{mL})$ of soluble PD-L1 in conditioned medium of SB cells after 24 hours of culture $(n=8)$. (J) Percentage of INF- $\gamma^{+} T$ cells and $\mathrm{Ki} \mathrm{7}^{+} \mathrm{T}$ cells after 24 hours of treatment with conditioned medium from SB cells ( $1 \mathrm{~mL}$ ) with IgG or anti-PD-L1 neutralizing antibody (SB-CM/IgG or SB-CM/anti$\mathrm{PD}-\mathrm{L} 1)$. T cells were isolated from WT mice $(n \geq 5)$. Data are represented as mean \pm SD. Unpaired Student's $t$ test (B and $\mathbf{G}-\mathbf{I}$ ) and 1-way ANOVA with Bonferroni's post hoc test (D-F and I) were used. ${ }^{*} P<0.05 ;{ }^{* *} P<0.01 ;{ }^{* *} P<0.001$.

extracellular vesicles (EVs) can induce a protumor immune microenvironment (27), we next assessed PD-L1 expression on SB cell-derived EVs. Although SB cell-derived EVs expressed PD-L1 (Supplemental Figure 2, D and E), incubating BMDMs with SB EVs did not alter macrophage-associated PD-L1 (Supplemental Figure 2F). PD-L1 may also be present in a circulating or soluble form with immunosuppressive properties that facilitate cancer progression $(28,29)$. Indeed, we identified soluble PD-L1 in conditioned medium from SB cells (Figure 2I), implying that soluble PD-L1 from CCA cells is transferred to TAMs. However, the percentage of soluble PD-L1 transferred to TAMs in our murine model appears to be trivial (Figure 2D). To assess whether soluble PD-L1 is functional, SB cell conditioned medium was incubated with anti-PD-L1 antibody to neutralize any soluble PD-L1, and conditioned medium was subsequently added to T cells. Neutralization of PD-L1 from the conditioned medium did not have an effect on T cell proliferation, as assessed by Ki67, or $\mathrm{T}$ cell activation, as assessed by IFN- $\gamma$ production (Figure 2J). 
A

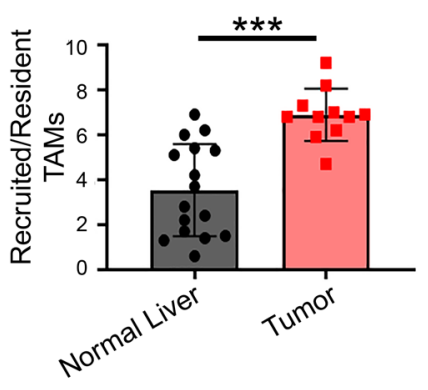

B $\quad \mathrm{PD}-\mathrm{L} 1+$ Recruited TAMs

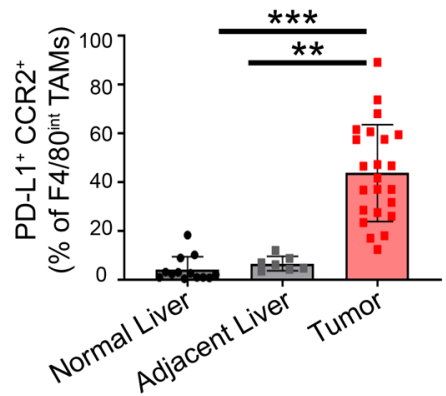

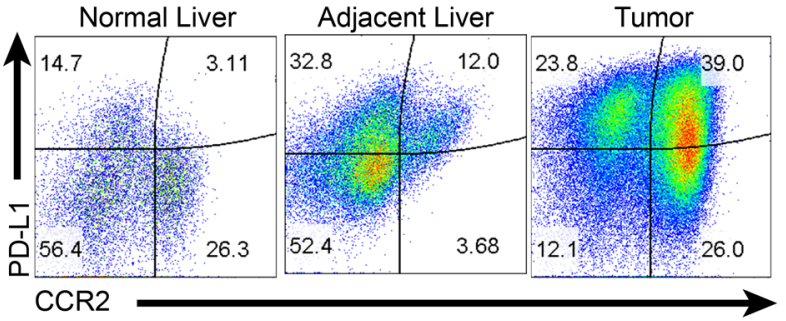

Gate on CD11b+F4/80 int TAMs
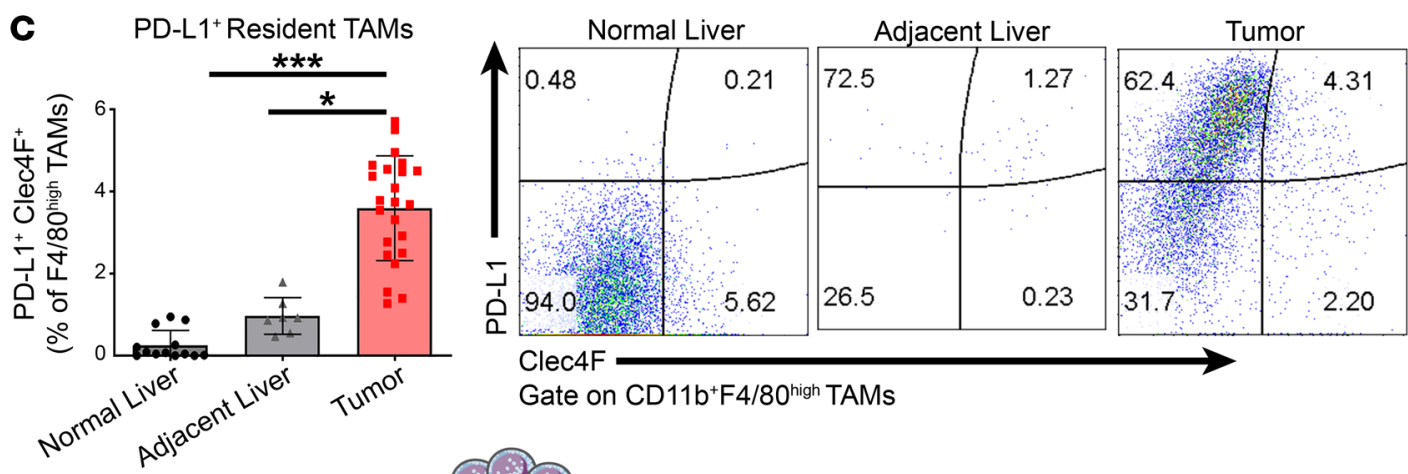

Gate on CD11b+F4/80 high TAMs
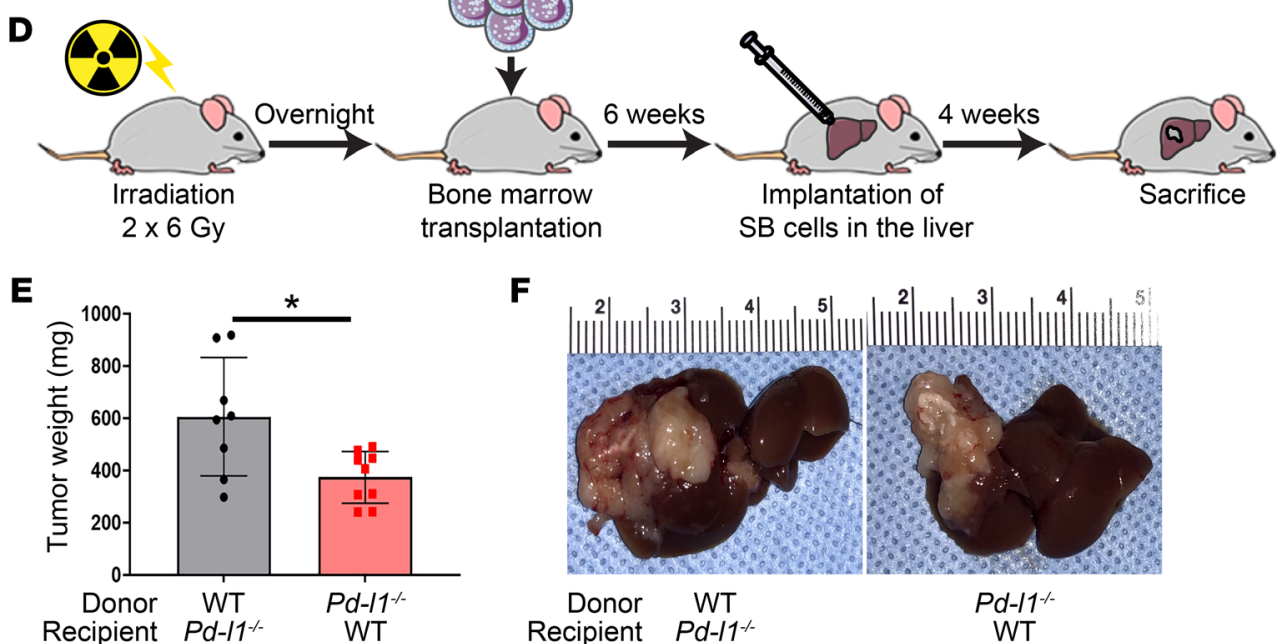

G

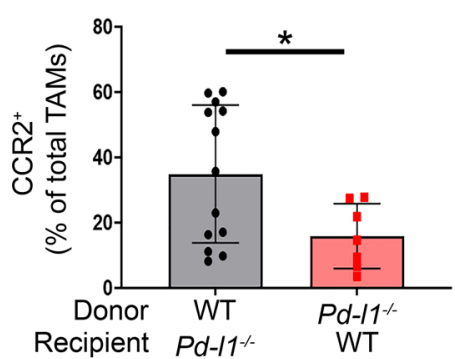

H

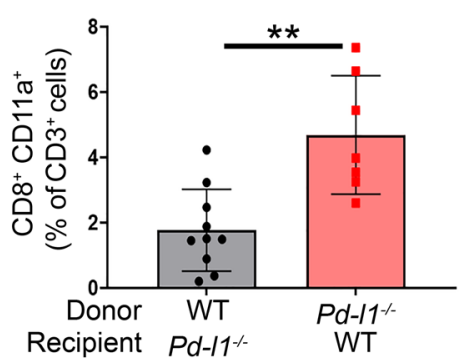

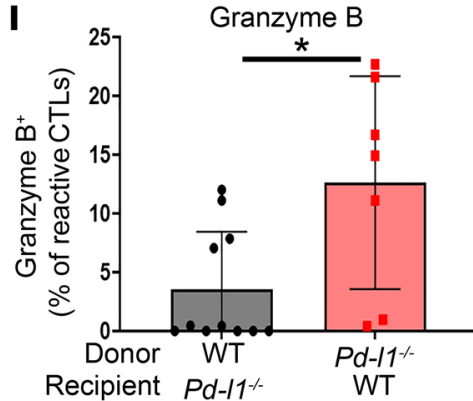

Figure 3. PD-L1+ TAMs are recruited from the BM in CCA. (A-C and E-I) Tumor growth of 28 days after orthotopic implantation of $1 \times 10^{6}$ SB (murine CCA) cells in

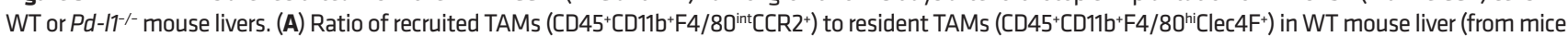
without tumors) or SB tumor ( $n \geq 11$ ). (B) Percentage of PD-L1+CCR2+ recruited TAMs of F4/80 int TAMs (CD45+CD11b+F4/80 int) in WT mouse liver, tumor-adjacent liver, or tumor. Representative flow plots show expression of CCR2 and PD-L1 in F4/80 int TAMs ( $n \geq 7$ ). (C) Percentage of PD-L1+Clec4F+ resident TAMs of F4/80 $0^{\text {hi }}$ TAMs $\left(C D 45^{+} C D 11 b^{+} F 4 / 80^{\text {hi }}\right.$ ) in WT mouse liver, tumor-adjacent liver, or tumor. Representative flow plots show expression of Clec $4 F$ and PD-L1 in F4/80 Schematic of mouse BM transplantation. (E) Average tumor weights in $\mathrm{mg}$ of $P d-11^{-/}$-mice transplanted with WT BM (WT-Pd-1 $1{ }^{-1-}$ ) or WT mice transplanted with $P d$ $17^{-1-} \mathrm{BM}\left(P d-11^{-1-}-\mathrm{WT}\right)(n \geq 8)$. (F) Representative photographs of livers from E. (C) Percentage of CCR2+ recruited TAMs of total TAMs (CD45+CD11b+F4/80+) in tumors

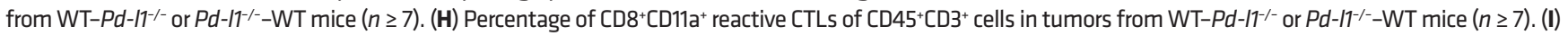

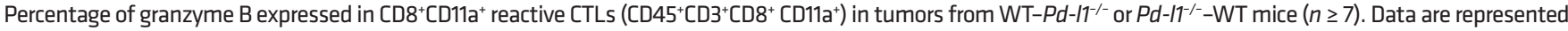
as mean \pm SD. Unpaired Student's $t$ test $(\mathbf{A}, \mathbf{E}$, and $\mathbf{G}-\mathbf{I})$ and 1-way ANOVA with Bonferroni's post hoc test (B and $\mathbf{C})$ were used. ${ }^{*} P<0.05 ;{ }^{*} P<0.01 ;{ }^{* * *} P<0.001$. 
Taken together, these results suggest that, although cancer cells transfer a small amount of PD-L1 to TAMs, this transferred soluble PD-L1 is not immunosuppressive based on its impact on $\mathrm{T}$ cells. As it is not functional, soluble PD-L1 likely does not contribute to CCA tumor biology in a meaningful manner.

$P D-L 1^{+} T A M$ s are recruited from the BM in CCA. Hepatic macrophages may be categorized as resident Kupffer cells (yolk sac derived) or recruited (from circulating monocytes differentiating into macrophages) $(30,31)$. The distinction between the two is important because strategies for blocking their protumor activity are different, as chemokine inhibitors may block macrophage recruitment to CCA, but would not alter resident Kupffer cell abundance or function. Although WT SB tumors had a significantly higher ratio of recruited macrophages $\left(\mathrm{CD} 45^{+} \mathrm{CD}\right.$ $\left.11 \mathrm{~b}^{+} \mathrm{F} 4 / 80^{+} \mathrm{CCR} 2^{+}\right)$than resident Kupffer cells $\left(\mathrm{CD} 45^{+} \mathrm{CD}-\right.$ $\left.11 \mathrm{~b}^{+} \mathrm{F} 4 / 80^{+} \mathrm{Clec} 4 \mathrm{~F}^{+}\right)$, PD-L1 was significantly increased in both subsets (Figure 3, A-C, and Supplemental Figure 3, A and B). Notably, the preponderance of resident macrophages was $\mathrm{F} 4 / 80^{\text {hi }}$, whereas recruited macrophages were F4/80 int (Supplemental Figure 3, C and D). Next, we sought to ascertain whether $\mathrm{PD}-\mathrm{L}^{+}$macrophages recruited from the BM promote CCA progression. We irradiated WT and $P d-l 1^{-/-}$mice and transplanted them with BM from $P d-l 1^{-/-}$and WT mice, respectively (Figure 3D and Supplemental Figure 3E). WT BM was transplanted into irradiated WT mice, and $\mathrm{Pd}-l 1^{-/-} \mathrm{BM}$ was transplanted into irradiated $\mathrm{Pd}-l 1^{-/-}$mice as a control (Supplemental Figure 3, E-G). $P d-l 1^{-/-}$mice with WT BM (WT-Pd-l1/-) had a significant increase in tumor burden compared with WT mice with $\mathrm{Pd}-\mathrm{l1}^{-1-}$ $\mathrm{BM}\left(\mathrm{Pd}-\mathrm{ll} \mathrm{1}^{-/-} \mathrm{WT}\right)$ (Figure 3, E and F). Accordingly, WT- $\mathrm{Pd}-\mathrm{ll} 1^{-1-}$ had an increase in recruited macrophages and decreased CD ${ }^{+}$ $\mathrm{T}$ cell infiltration. In contrast, $\mathrm{Pd}-\mathrm{l} 1^{-/-} \mathrm{WT}$ mice had a significant decrease in recruited macrophages and increased $\mathrm{CD}^{+} \mathrm{T}$ cell activation and effector function (Figure 3, G-I). Taken together, these data suggest that recruited macrophages are the primary source of $\mathrm{PD}-\mathrm{L}^{+}$macrophages, and facilitate CCA progression.

TAM blockade promotes a compensatory accumulation of G-MDSCs. Recruitment of infiltrating monocyte-derived macrophages to the tumor bed in malignancies is dependent on CCR2 (32). Accordingly, Ccr2-deficient mice have defective monocyte recruitment (33). As we had observed an increase in recruited TAMs in WT tumors, we hypothesized that the $\mathrm{Ccr} 2^{-/-}$mice would have reduced tumor burden. Unexpectedly, $\mathrm{Ccr} 2^{--}$mice had tumor burden and $\mathrm{CD}^{+} \mathrm{T}$ cell infiltration similar to that of WT mice following SB cell implantation (Figure 4A and Supplemental Figure 4, A-C). As expected, $\mathrm{Crr}^{-/-}$mice did not have PD-L1 ${ }^{+}$ recruited TAMs (Supplemental Figure 4D). However, an increase in $\mathrm{PD}-\mathrm{L}^{+}$resident TAMs was not observed either (Figure 4B), suggesting compensatory emergence of another immunosuppressive cell population. MDSCs, immature myeloid cells with robust immunosuppressive properties, mediate tumor immune evasion and immunotherapy resistance $(34,35)$. Using flow cytometry, we demonstrated an increase in $\mathrm{CD}_{11} \mathrm{~b}^{+} \mathrm{Gr}-1^{+}$MDSCs in $\mathrm{Ccr} 2^{-/-}$mice compared with WT mice (Figure 4C). In mice, MDSCs encompass CD11b ${ }^{+}$Gr- $1^{\text {hi }}\left(\right.$ Ly6C ${ }^{\text {lo }}$ Ly6G $\left.{ }^{\text {hi }}\right)$ G-MDSCs and CD11b ${ }^{+}$Gr- $1^{\text {int }}\left(\right.$ Ly6C ${ }^{\text {h- }}$ ${ }^{\mathrm{i}} \mathrm{Ly} 6 \mathrm{G}^{\mathrm{lo}}$ ) monocytic MDSCs (M-MDSCs) (36). Further characterization of MDSCs revealed that G-MDSCs were the dominant subset in $\mathrm{Ccr}^{-/-}$mice (Figure 4D).
To test the effect of pharmacologic TAM inhibition on CCA tumor growth, CSF1R inhibition was employed (Figure 4E). The efficiency of TAM inhibition with anti-CSF1R was confirmed (Supplemental Figure 4, E and F). However, CSF1R inhibition also did not reduce CCA growth in mice (Figure 4F and Supplemental Figure 4, G-I). Mass cytometry analysis of the CSF1R-treated SB tumors revealed a compensatory infiltration of Ly $6 C^{\mathrm{lo}} \mathrm{Ly} 6 \mathrm{G}^{\mathrm{hi}}$ G-MDSCs (Figure 4, G-I). Accordingly, reactive $\mathrm{CD}^{+} \mathrm{T}$ cells were unchanged between the vehicle and CSF1R-treated tumors (Supplemental Figure 4J). These observations highlight that prevention of TAM recruitment or pharmacologic TAM inhibition promotes a compensatory infiltration of G-MDSCs and counteracts the potential antitumor effect of eliminating protumor macrophages in murine CCA.

CAF-derived CXCL2 is increased in the context of TAM blockade. To examine the potential mechanism of TAM blockade-mediated G-MDSC accumulation in tumors, we assessed the expression of various chemokines in control and anti-CSF1R-treated tumors using an unbiased screen. A significant upregulation of several chemokines implicated in MDSC recruitment was observed in the anti-CSF1R-treated tumors compared with control-treated tumors (Supplemental Figure 5A). Expression of potential chemokines identified by the chemokine array was further assessed using quantitative PCR in multiple samples from vehicle and anti-CSF1R-treated tumors (Figure 5A and Supplemental Figure 5B). Cxcl2 was significantly upregulated in the anti-CSF1R-treated tumors compared with vehicle-treated tumors (Figure 5A). As $\mathrm{Cxcl} 2$ is a known chemoattractant for MDSCs (37), we conducted additional studies to investigate the cellular source of this chemokine. Using FISH, we demonstrated a significant increase in $\mathrm{Cxcl} 2$ in SB tumors compared with adjacent liver (Supplemental Figure $5 C)$. Consistent with this observation, MDSCs were increased in SB tumors compared with adjacent liver (Supplemental Figure 5D). Notably, anti-CSF1R-treated murine tumors had a significantly higher expression of $\mathrm{Cxcl} 2$ compared with vehicle-treated murine tumors (Figure $5 \mathrm{~B}$ ). $\alpha$-Smooth muscle actin-positive ( $\alpha$-SMA-positive) cancer-associated fibroblasts (CAFs), rather than the CCA cells, appeared to be the primary source of $\mathrm{Cxcl} 2$ in anti-CSF1R-treated tumors (Figure 5B). Accordingly, anti-CSF1R-treated tumors had an increased abundance of CAFs compared with vehicle-treated tumors (Figure 5C). These observations are consistent with prior work demonstrating that CAFs increase recruitment of MDSCs to anti-CSF1R-treated tumors via the CXCL1/CXCR2 axis in murine lung carcinoma and lymphoma (38). In aggregate, these results suggest that TAM blockade employing CSF1R inhibition is associated with increased production of CXCL2 by CAFs with consequent enhanced G-MDSC accumulation in tumors.

Single-cell transcriptomics demonstrates accumulation of G-MDSC subsets with an immunosuppressive signature in the context of TAM blockade. To characterize the phenotype of anti-CSF1R-induced G-MDSC infiltration in CCA, we isolated CD11b ${ }^{+}$Ly6 ${ }^{\mathrm{lo}} \mathrm{Ly} 6 \mathrm{G}^{\mathrm{Hi}}$ G-MDSCs from SB tumors of vehicle or anti-CSF1R-treated mice via FACS (Figure 6A). We then employed a droplet-mediated single-cell RNA sequencing (scRNA-Seq) platform to profile FACS-purified live G-MDSCs. We profiled 2 tumor samples from vehicle-treated mice $(4,757$ 

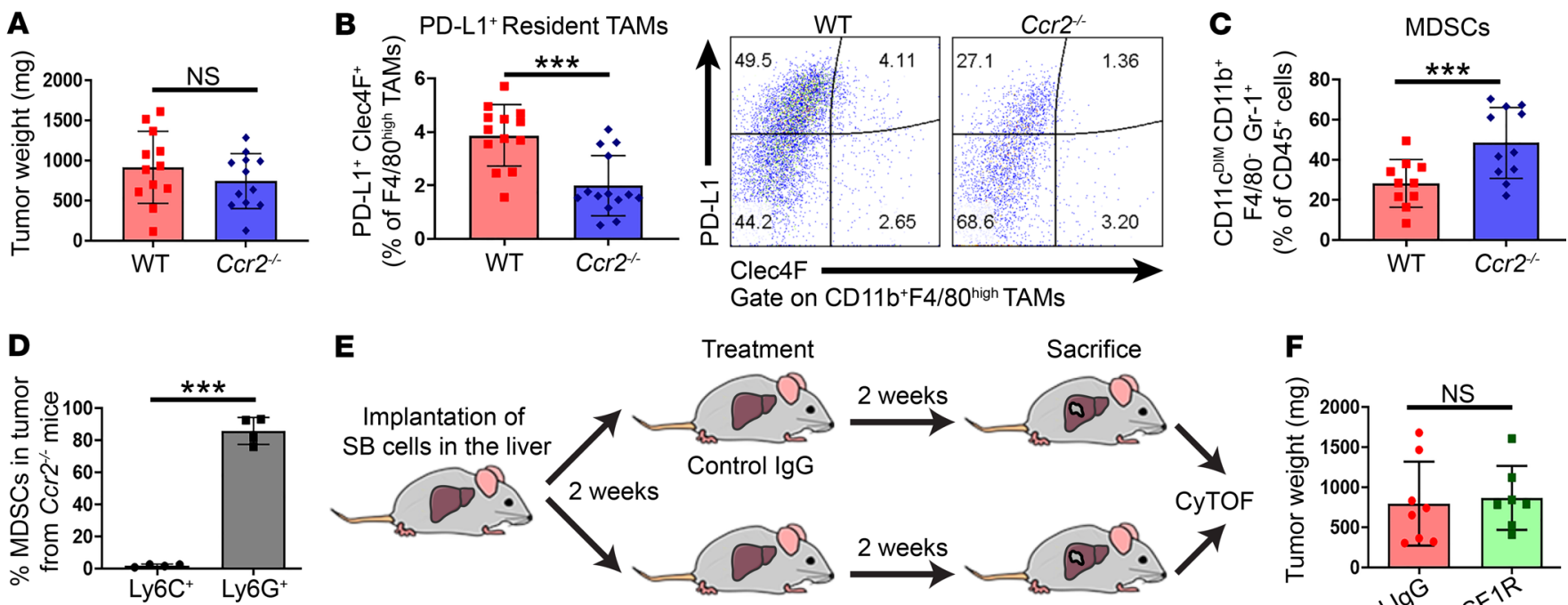

\section{E}
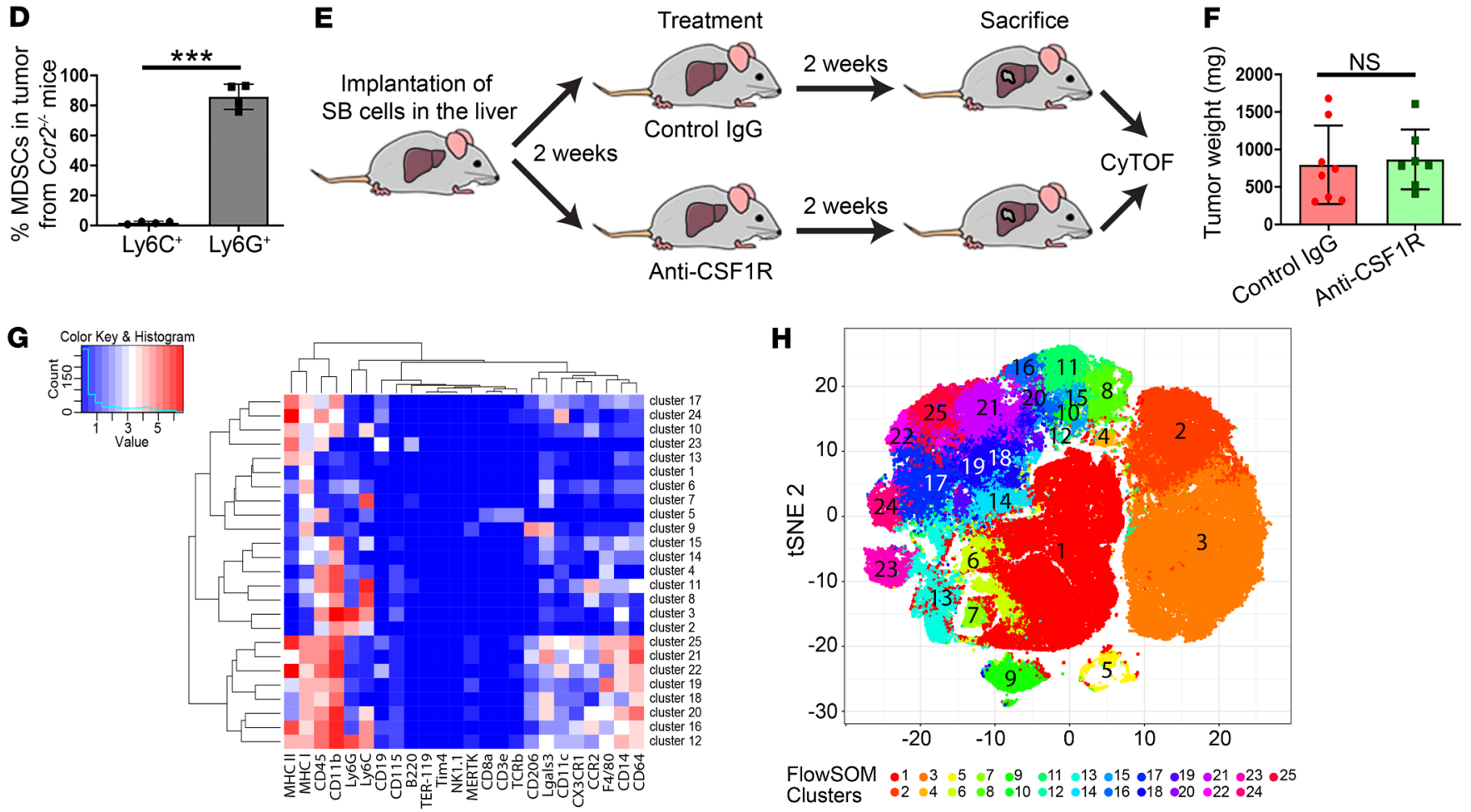

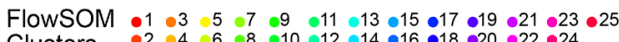
Clusters $\bullet 2 \quad 4 \quad 6 \quad 8 \quad \bullet 10 \cdot 12 \bullet 14 \bullet 16 \bullet 18 \bullet 20 \bullet 22 \bullet 24$
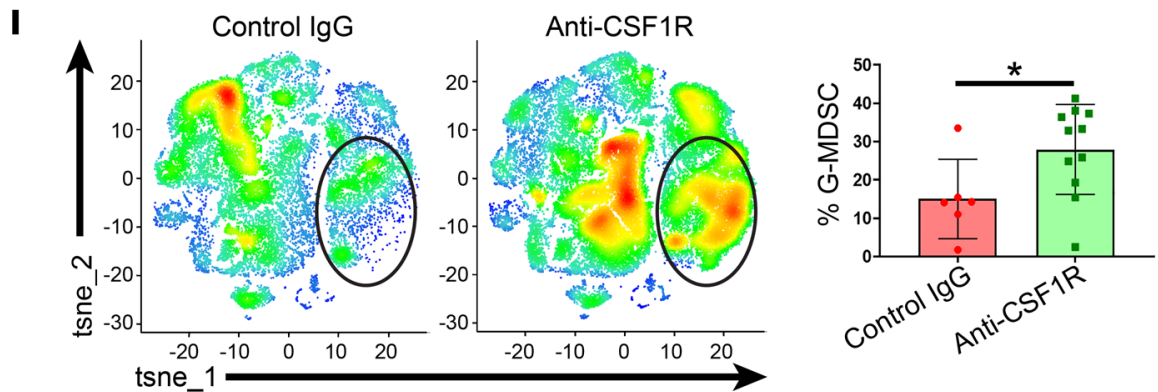

Figure 4. TAM blockade promotes a compensatory infiltration of G-MDSCs. (A-F and I) Tumor growth of 28 days after orthotopic implantation of $1 \times 10^{6} \mathrm{SB}$ (murine CCA) cells in WT or C $\mathrm{Cr} 2^{-/-}$mouse livers. (A) Average tumor weights in $\mathrm{mg}$ of WT and $C \mathrm{cr} 2^{-/-}$mice $(n=12)$. (B) Percentage of

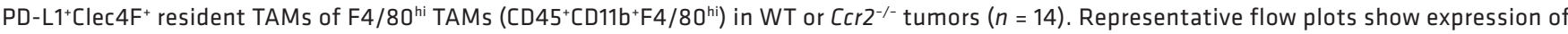

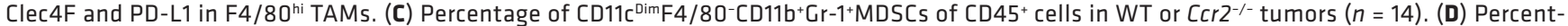

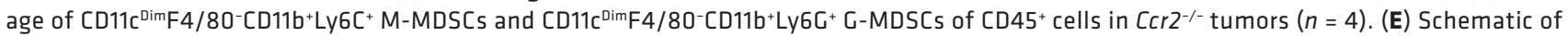
myeloid cell analysis in anti-CSF1R- and control-treated mouse tumors. (F) Average tumor weights in mg of WT mice treated every 3 days from days 14 to 28 (after orthotopic SB cell implantation) with a control rat IgG isotype or anti-mouse CSF1R (AFS98) ( $n \geq 7$ ). (G) Heatmap showing average marker expression intensity in the different CyTOF clusters. (H) tSNE plots of CyTOF data sets show different clusters of immune cell populations identified by selected markers. (I) tSNE plots of CyTOF data sets show different clusters of immune cell populations identified by selected markers in tumor from WT mice treated with control IgG $(n=6)$ or anti-CSF1R $(n=11)$. Cells are color coded and represent the mean of cell density in each cluster. Black circles outline the G-MDSC cluster (cluster 3). Percentage of G-MDSCs identified by markers expressed in cluster 3 in tumor from WT mice treated with control IgG or anti-CSF1R $(n \geq 6)$. Data are represented as mean \pm SD. One-way ANOVA with Bonferroni's post hoc test was used. ${ }^{*} P<0.05 ;{ }^{* *} P<0.001$. 
A

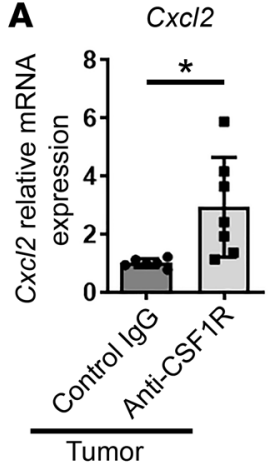

B

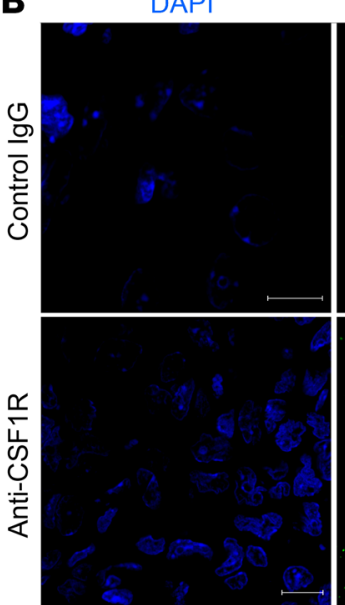

DAPI

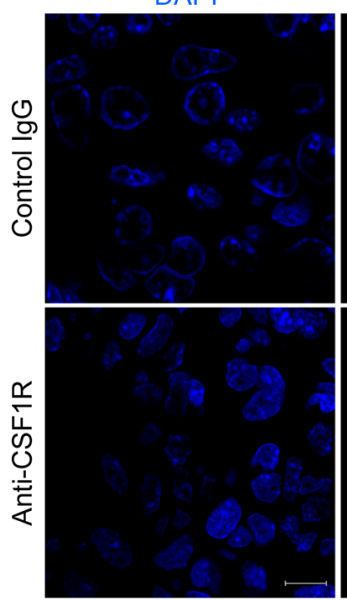

a-SMA
CXCL2

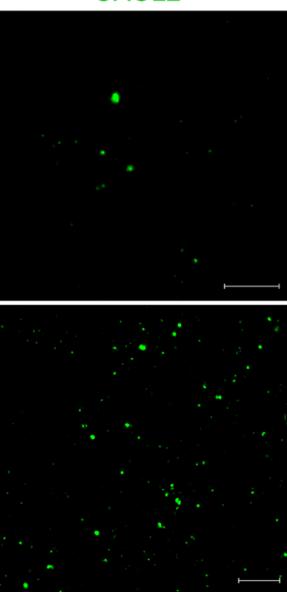

CXCL2

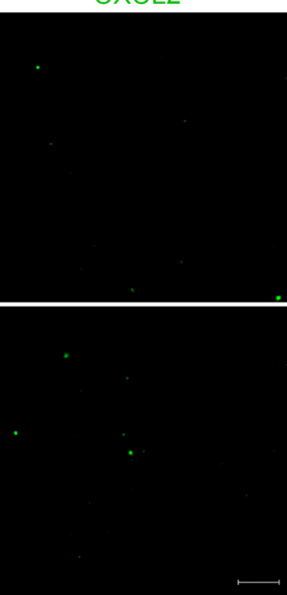

a-SMA

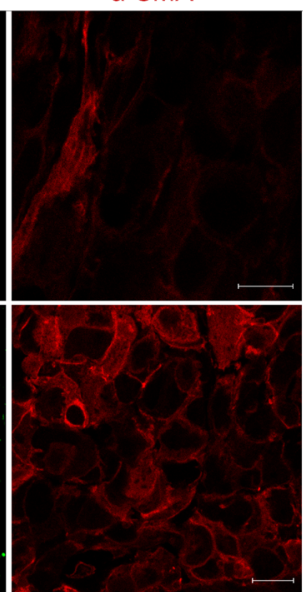

CK19

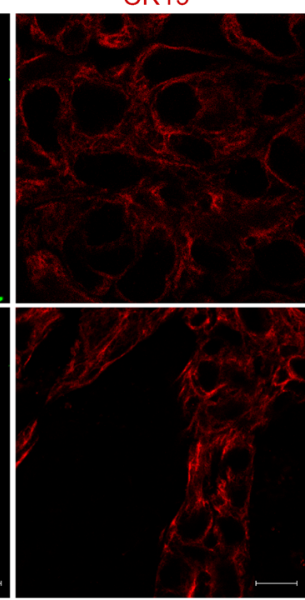

Merge
C

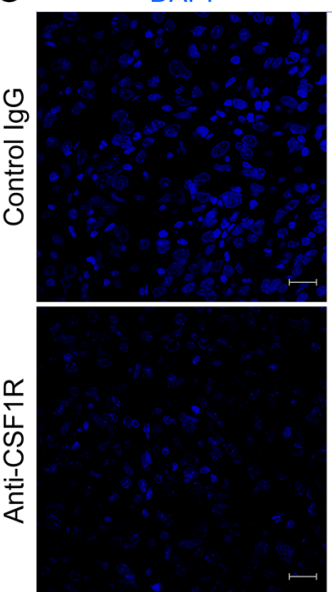

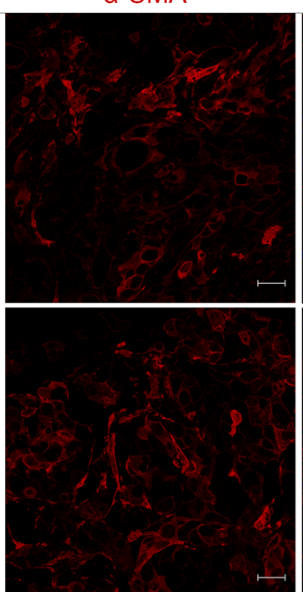
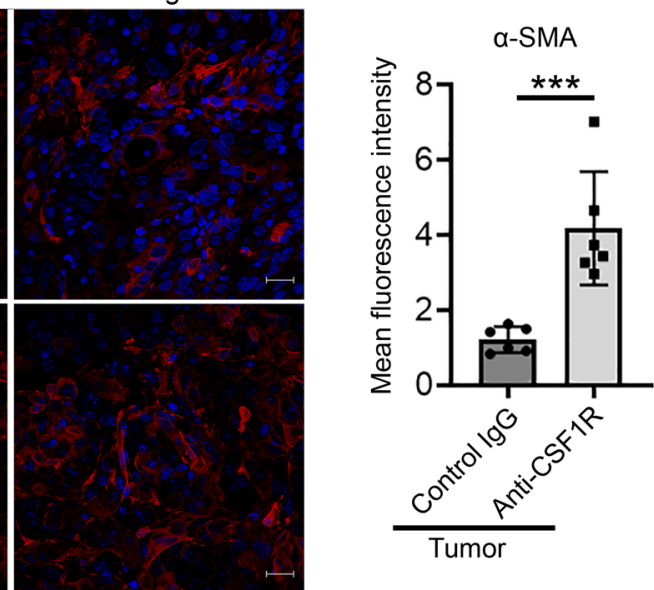

Figure 5. CAF-derived CXCL2 is increased in the context of TAM blockade. (A-C) Tumor growth of 28 days after orthotopic implantation of $1 \times 10^{6}$ SB (murine CCA) cells in WT mouse livers. (A) Relative mRNA expression of Cxcl2 in control or anti-CSF1R-treated SB tumors ( $n \geq 6$ ). (B) Representative immunofluorescence images of $\alpha$-SMA (upper panels) or CK-19 (lower panels) in red, Cxcl2 by in situ hybridization in green, and nuclei counterstained with DAPI in control or anti-CSF1R-treated mouse tumor. Scale bars: $10 \mu \mathrm{m}$. (C) Representative immunofluorescence images of $\alpha$-SMA (left panel) in red, counterstained nuclei with DAPI in control or anti-CSF1R treated-mouse tumor. Scale bars: $20 \mu \mathrm{m}$. Quantification of mean fluorescence intensity of $\alpha$-SMA signal in control or anti-CSF1R-treated mouse liver (right panel). Data are represented as mean \pm SD. Unpaired Student's $t$ test was used. ${ }^{*} P<0.05$; ${ }^{* *} P<0.001$.

cells) and anti-CSF1R-treated mice (6480 cells), respectively, for a total of 11,237 cells. We initially identified 8 clusters in the vehicle and anti-CSF1R-treated samples with high resolution (resolution $=0.5)($ Figure 6B). However, after further review of expression profiles for each cluster of cells, clusters with similar gene-expressed profiles were combined and ultimately there were 2 distinct clusters (cluster 0 and 1 , resolution $=0.01$ ) of G-MDSCs in the murine tumors, with substantial differ- 
ences in transcriptomic activity (Figure 6C and Supplemental Table 1 and Supplemental Table 2). Although cluster 0 slightly decreased in cell abundance with anti-CSF1R treatment, cluster 1 was significantly enriched in the anti-CSF1R-treated tumor sample (Figure 6C and Supplemental Figure 6A). Cluster $\mathrm{O}$ was characterized by higher expression of MDSC-related genes and did not have significant expression of neutrophil- or monocyte-related genes (Supplemental Figure 6B and Supplemental Table 1). Cluster 1 was associated with expression of MDSC-related genes, but also displayed expression of a few monocyte-related genes and, to a lesser extent, neutrophil-related genes, suggesting that this cluster may be less well differentiated or more immature than cluster 0 (ref. 39 and Supplemental Figure 6B and Supplemental Table 2).

scRNA-Seq analysis uncovers a distinct ApoE G-MDSC subset. Apoe, a transcriptional target of liver-X receptors (LXR), was the top conserved gene in cluster 1 and was also significantly downregulated with anti-CSF1R treatment (Figure 6D and Supplemental Figure 7B). In contrast, cluster 0 or classic G-MDSCs did not display expression of ApoE (Figure 6D). ApoE is a secreted protein implicated in lipoprotein metabolism and inhibition of metastatic progression in melanoma (40). More recently, the $\mathrm{LXR} / \mathrm{ApoE}$ axis has been linked to MDSC depletion via enhanced apoptosis across several tumor types (36). Notably, there was significant cell enrichment of cluster 1 or ApoE G-MDSCs with anti-CSF1R treatment $(P<0.01)$ (Supplemental Figure 6A), suggesting that perhaps this cluster has survival capabilities, as evidenced by significant downregulation of Apoe $(P<0.001)$ (Figure 7A). Moreover, ApoE G-MDSCs displayed an immunosuppressive gene signature (Figure 6D) with enhanced expression of several genes implicated in MDSC-related immunosuppression, tumorigenesis, and metastasis, including Arginase 1 (Arg1), S100 calcium binding protein A4 (S1OOa4), CD74 antigen (Cd74), and Peroxiredoxin 1 (Prdx1) $(16,41)$.

To determine the human relevance of the ApoE G-MDSC subpopulation, we compared murine ApoE G-MDSC signature genes with a human scRNA-Seq data set comprising 10 human CCAs and 9 hepatocellular carcinomas (HCCs) (GSE125449) (42). Analysis of the single-cell transcriptomic profile of the CCA patients in this data set revealed 8 broad predicted cell types, including TAMs, CAFs, malignant cells, and T cells (Supplemental Figure 6C and ref. 42). The cell population predicted as TAMs likely included other myeloid cells, such as MDSCs, as MDSCs were not distinctly categorized in the original cell-type prediction (42). Accordingly, we conducted an AUCell gene set enrichment analysis to assess for the presence of MDSCs in human CCA. A set of known human MDSC signature genes $(43,44)$ was enriched in the cell population predicted as TAMs and, to a lesser extent, in the cell population predicted as $\mathrm{T}$ cells by Ma et al. (ref. 42, Figure 6E, and Supplemental Table 3). Next, we conducted a second AUCell gene set enrichment analysis to assess for the presence of a cell population similar to ApoE G-MDSCs in human CCA. ApoE G-MDSC signature genes from our murine model (Supplemental Table 4) were significantly enriched in the subset of CCA cells predicted as TAMs (Figure 6F). Moreover, a subset of these cells had abundant ApoE expression (Supplemental Figure 6D). These results imply that ApoE G-MDSCs comprise a unique MDSC subset that is present in human CCA.
TAM blockade facilitates emergence of G-MDSC subsets with immunosuppressive properties. The accumulation of G-MDSCs in the context of TAM blockade may be due to enhanced survival and/or increased recruitment. ApoE G-MDSCs in the anti-CSF1Rtreated tumors had downregulation of genes implicated in MDSC cell death. Apoe as well as cathepsin D (ctsd) and cathepsin B (ctsb), which mediate MDSC death via interrupted autophagy and endoplasmic reticulum stress, were downregulated $(36,45)$. Conversely, S1OOa4, which is essential for MDSC survival, was significantly upregulated (ref. 41, Figure 7A, and Supplemental Figure 7, A and B). To ascertain the functional relevance of these observations, we assessed the impact of TAM blockade on G-MDSC apoptosis. We observed a significant decrease in the percentage of G-MDSCs from anti-CSF1R-treated tumors staining positive for annexin $\mathrm{V}$ and 7-amino-actinmycin D compared with G-MDSCs from vehicle-treated tumors (Figure 7B).

Activation of STAT1 as well as NF- $\kappa \mathrm{B}$ promotes MDSC activation, with consequent upregulation of arginase 1 and inducible nitric oxide synthase (46). The IFN- $\gamma /$ STAT1 axis fosters MDSC suppressive activity (16). Interestingly, anti-CSF1R treatment was associated with a significant upregulation of Stat1 in G-MDSCs, suggesting MDSC activation and enhanced suppressive activity (Figure 7C). Moreover, G-MDSCs displayed significant downreg-

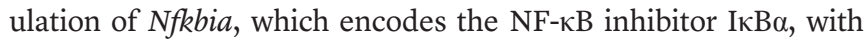
anti-CSF1R treatment (Figure 7C and Supplemental Figure 7, A and B). We next determined suppressive properties of G-MDSCs in the anti-CSF1R-treated tumors. Compared with G-MDSCs isolated from vehicle tumors, G-MDSCs isolated from anti-CSF1R-treated tumors exhibited more significant suppression of $\mathrm{CD}^{+} \mathrm{T}$ cell proliferation, as assessed by Ki67, as well as $\mathrm{CD}^{+} \mathrm{T}$ cell activation, as assessed by IFN- $\gamma$ production (Figure 7D). In summary, these findings suggest that TAM blockade is associated with emergence of G-MDSCs with immunosuppressive signatures and function.

G-MDSC sinteract with CTLs in human CCA. Next, we assessed the interplay between G-MDSCs and T lymphocytes in human CCA. Using imaging mass cytometry, we identified cell-cell con-

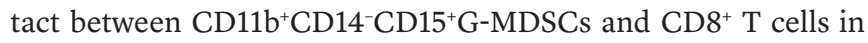
resected human CCA specimens (Figure 7E and Supplemental Figure 7C). Moreover, human CCAs displayed a poorly immunogenic TIME (8), with the tumor core being relatively devoid of $\mathrm{T}$ cells (Supplemental Figure 7D). T cells were present along the tumor margin intermingled with $\mathrm{CD}_{11} \mathrm{~b}^{+}$myeloid cells (Supplemental Figure 7D), implying that immunosuppressive myeloid cells may prevent $\mathrm{T}$ lymphocyte infiltration into the tumor core. Moreover, similarly to the results in our murine model, the majority of the myeloid cells expressed PD-L1, and the CD8 ${ }^{+} \mathrm{T}$ cells expressed PD-1 (Supplemental Figure 7, E and F). Finally, we assessed for the presence of G-MDSCs in 5 patients undergoing surgical resection; 2 patients had received neoadjuvant chemoradiation with gemcitabine and cisplatin. Flow cytometry was conducted on human CCA tumor immediately following surgical resection. $\mathrm{CD} 11 \mathrm{~b}^{+} \mathrm{CD} 14^{-} \mathrm{CD} 15^{+} \mathrm{G}-\mathrm{MDSCs}$ were detected in all 5 patients (Figure 7F).

Dual inhibition of G-MDSCs and TAMs potentiates anti-PD-1 therapy. As G-MDSCs counteract the effect of TAM-directed therapy by mediating tumor evasion, we hypothesized that dual inhibition of G-MDSCs and TAMs may have therapeutic bene- 
A
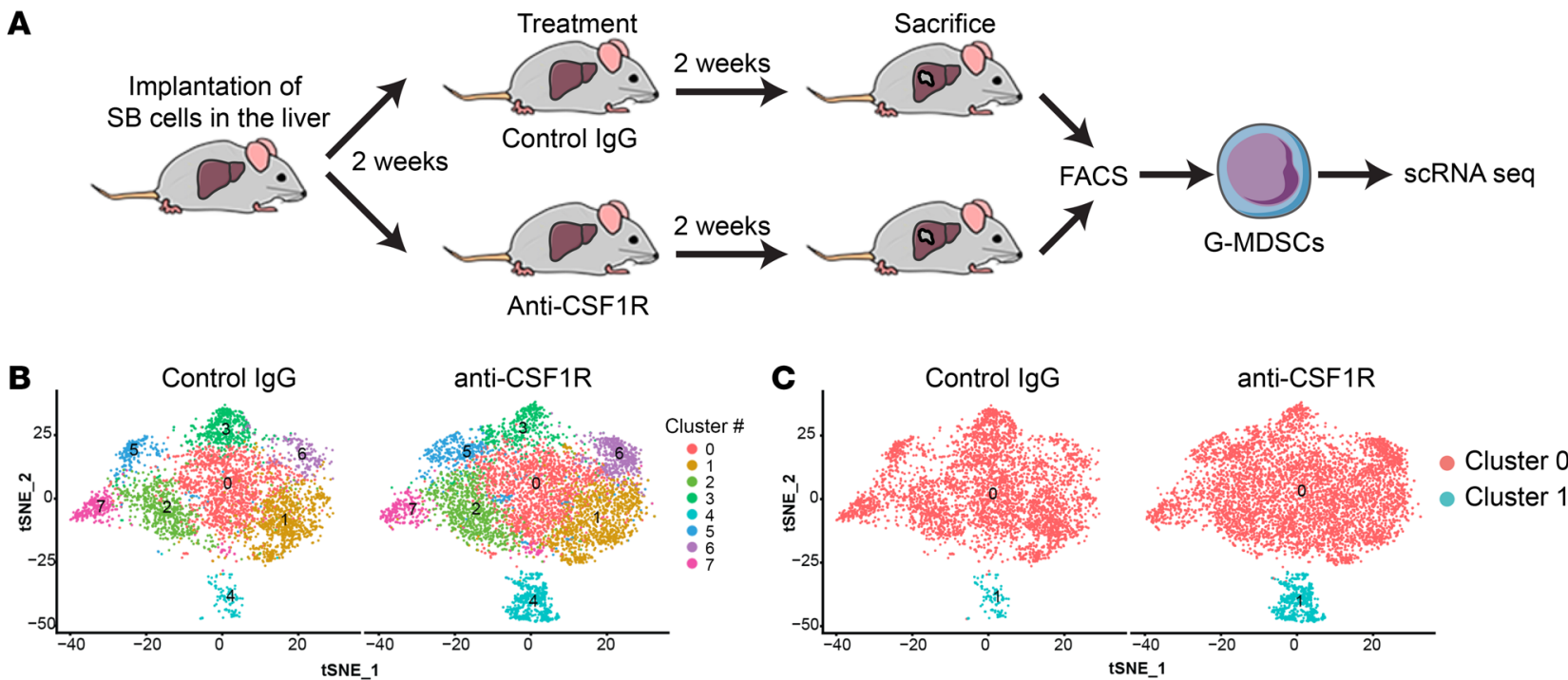

D

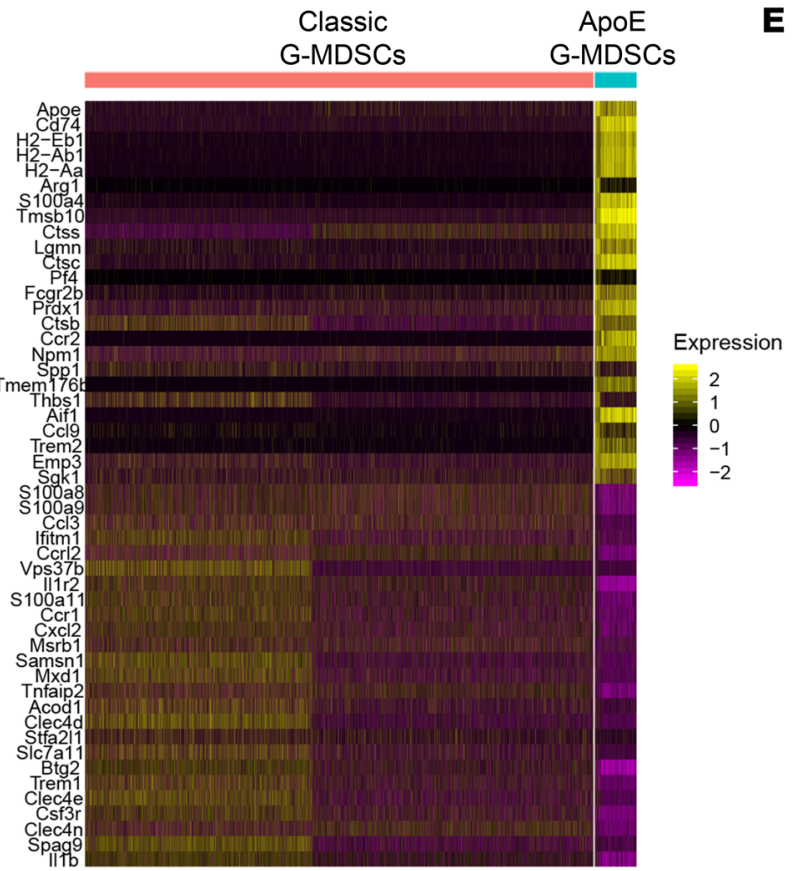

$\mathbf{E}$

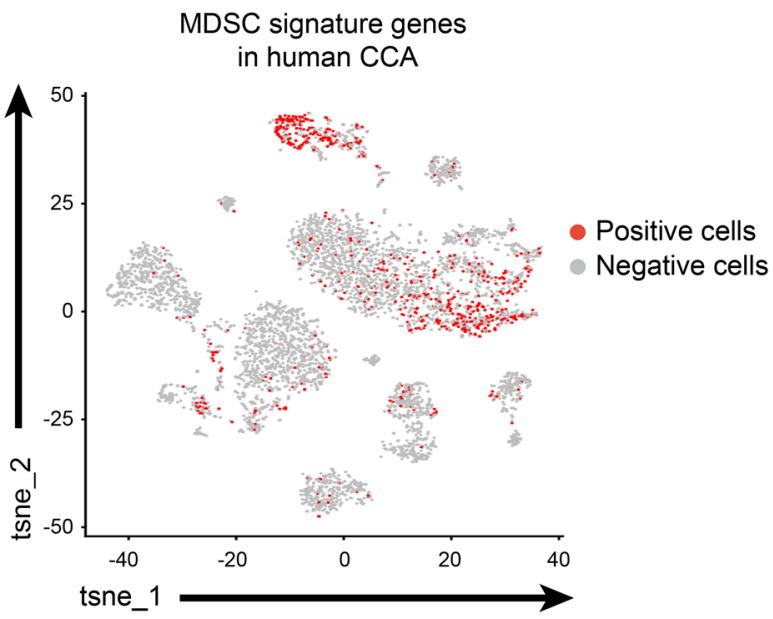

F ApoE G-MDSC signature genes

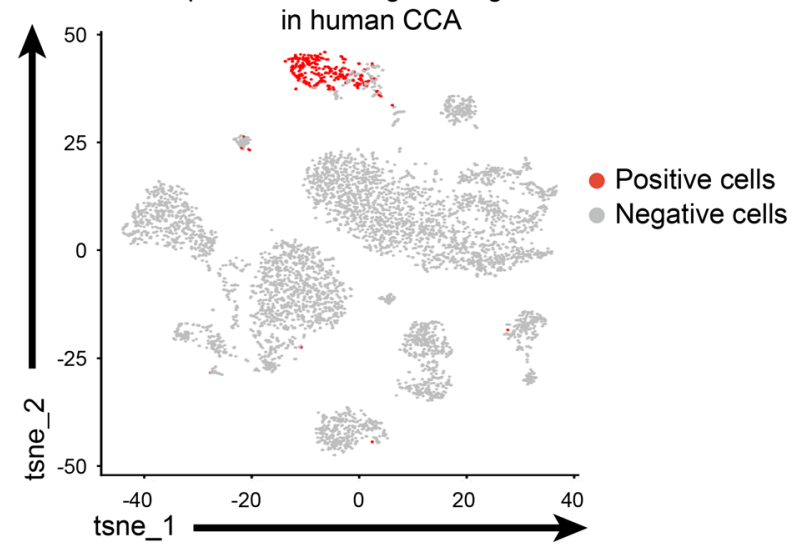

Figure 6. Single-cell transcriptomics demonstrates accumulation of unique G-MDSC subsets with TAM blockade. (A) Schematic depicting scRNA-Seq study of FACS-sorted G-MDSCs from control and anti-CSF1Rtreated murine tumors. (B and C) Tumor growth of 28 days after orthotopic implantation of $1 \times 10^{6} \mathrm{SB}$ (murine CCA) cells in WT mice. Mice were treated from day 14 to day 28 after implantation with control rat IgC isotype or anti-CSF1R (AFS98). (B) Cell clustering based on tSNE algorithm for WT mouse samples treated with control IgG or anti-CSF1R. Eight clusters were initially identified with high resolution (resolution $=0.5$ ) based on a shared nearest neighbor clustering algorithm as implemented in Seurat. (C) Cell clusters with similar expression profiles were further combined with resultant 2 distinct cell clusters. Percentage of cells in cluster 0 was $98 \%$ for control sample and $86 \%$ for anti-CSF1R sample. $P<0.01$, Fisher's exact test. (D) Heatmap of gene expression profiles for selected top cluster-specific genes ( $n=25$ for cluster 0 and cluster 1$)$. Expression values for each gene were $Z$ scored across all cells. (E) Enrichment analysis for 40 signature human MDSC genes using AUCell in human CCA $(n=10)$. Significantly enriched cells are highlighted in red. (F) Enrichment analysis for $40 \mathrm{ApoE}$ G-MDSC signature genes using AUCell in human CCA $(n=10)$. Significantly enriched cells are highlighted in red. 
fit in murine CCA. For therapeutic targeting of G-MDSCs, we employed the G-MDSC-specific anti-Ly6G antibody (clone 1A8). Flow cytometry confirmed a significant reduction of tumor as well as splenic G-MDSCs in anti-Ly6G-treated mice (Supplemental Figure 8, A and B). The combination of G-MDSC inhibition with anti-Ly6G and TAM inhibition using anti-CSF1R potentiated ICB using anti-PD-1. Anti-PD-1+anti-CSF1R+anti-Ly6G significantly prolonged the survival of mice bearing $S B$ tumors compared with anti-PD-1+anti-CSF1R or anti-PD-1+anti-Ly6G (Figure 8A). Tumor-bearing mice were followed with cross-sectional imaging using micro-CT while undergoing drug treatment (Figure 8C). Micro-CT imaging demonstrated significant improvement in tumor burden in anti-Ly6G+anti-CSF1R+anti-PD-1, which was confirmed at the time of sacrifice (Figure 8, B-D, and Supplemental Figure 8, C and D). Thus far, ICB monotherapy in human clinical trials of CCA has been disappointing $(5.8 \%$ objective response rate with the anti-PD-1 antibody pembrolizumab) (7). Anti-PD-1 therapy alone failed to influence survival of mice with CCA or reduce the tumor burden, and these results in mice are consistent with the experience in the human disease (Figure 8A). Anti-Ly6G treatment alone also did not affect murine survival (Figure 8A).

The LXR/ApoE axis reduces MDSC abundance in solid tumors via enhanced apoptosis (36). Pharmacologic activation of LXR using the agonist GW3965 has been shown to reduce MDSC abundance and significantly suppress tumor growth across several malignancies (7). Accordingly, we assessed GW3965 in combination with TAM blockade and ICB and observed a significant reduction in tumor burden in GW3965+anti-CSF1R+anti-PD-1-treated tumors (Figure 8D). GW3965 monotherapy has a tumor-suppressive effect in several malignancies, including ovarian cancer, glioblastoma, and renal cell carcinoma (36). However, in our murine CCA model, GW3965 monotherapy did not reduce tumor burden, suggesting that combined inhibition of MDSCs and TAMs is required for a tumor-suppressive effect in CCA (Supplemental Figure 8, E and F). Further characterization of the anti-Ly6G+anti-CSF1R+antiPD-1-treated SB tumors and the GW3965+anti-CSF1R+anti-PD-1treated SB tumors demonstrated a significant reduction in $\mathrm{PD}-\mathrm{L}^{+}$ TAMs and G-MDSCs compared with control antibody-treated tumors (Figure 8, E and $\mathrm{F}$ ). The alteration of the innate immune landscape of murine CCA tumors by anti-Ly6G+anti-CSF1R+antiPD-1 as well as GW3965+anti-CSF1R+anti-PD-1 therapy resulted in an increase in $\mathrm{CD}^{+} \mathrm{T}$ cell infiltration and activation as well as increased effector function (Figure 8, G-I). In summary, we have demonstrated that dual inhibition of TAMs and G-MDSCs is necessary to potentiate ICB employing anti-PD-1 with a substantial tumor-suppressive effect and improvement in murine survival.

\section{Discussion}

In examining the immunobiology of desmoplastic malignancies such as CCA, we have identified an essential role of immunosuppressive myeloid cells in tumor progression. These data indicate that (a) macrophages are the primary source of PD-L1 in murine and human CCA; (b) PD-L1+ TAMs mediate a protumor immune response with consequent tumor progression; (c) compensatory accumulation of G-MDSC subsets neutralizes the potential antitumor effect of TAM targeted therapy; (d) a unique ApoE G-MDSC subset in murine and human CCA has immunosuppressive properties; and (e) dual inhibition of G-MDSCs and TAMs is necessary to potentiate anti-PD-1 therapy in CCA. These results are further discussed below.

Immunotherapy has revolutionized the treatment of human cancers, with a subset of patients across a variety of malignancies having durable responses to ICB targeting PD-1 or PD-L1. However, the response rate to ICB has been disappointing in other cancer types such as CCA (response rate of only $5.8 \%$ to ICB monotherapy) (7). TIME phenotype affects ICB response with a poorly immunogenic or "cold" TIME correlating with a poor response rate. This phenotype is populated by immunosuppressive innate immune cells such as TAMs and MDSCs. Our study reveals that murine CCAs have an abundance of these immunosuppressive elements, particularly TAMs. Notably, we find that TAMs are the predominant source of PD-L1 in human and murine CCA. Implantation of murine CCA cells in Pd-l1-deficient mice resulted in enhanced $\mathrm{CD}^{+} \mathrm{T}$ cell infiltration and activation with consequent marked reduction in tumor size compared with WT mice, indicating that PD-L1+ host myeloid cells, predominantly TAMs, foster a protumor TIME that facilitates CCA progression.

Hepatic macrophages may be categorized as resident Kupffer cells (yolk sac derived) (30) or recruited (30,31). Our study has found that recruited macrophages make up a substantial component of the protumor macrophage subset in our murine model of CCA and foster tumor growth in established CCA. Interestingly, $\mathrm{Ccr}^{-/-}$mice that are devoid of recruited macrophages had a tumor burden similar to that of WT mice, likely due to a compensatory increase of immunosuppressive G-MDSCs. TAM blockade in murine CCA tumors using anti-CSF1R also did not delay tumor progression despite a marked reduction in $\mathrm{CD} 11 \mathrm{~b}^{+} \mathrm{F} 4 / 80^{+} \mathrm{Gr}-1^{-}$ macrophages. Similarly to the results in the $\mathrm{C} c 2^{-/-}$mice, inhibition of CSF1R signaling also induced a robust accumulation of G-MDSCs in SB tumors. Expression of Cxcl2, a known G-MDSC chemoattractant, was substantially upregulated in anti-CSF1Rtreated tumors compared with control tumors. CAFs appeared to be the primary source of $\mathrm{Cxcl} 2$ in the CCA TIME. Accordingly, an increased abundance of CAFs was noted in the context of TAM blockade. These observations are consistent with a prior study that identified enhanced MDSC recruitment to anti-CSF1R-treated murine lung and lymphoma tumors as a consequence of chemokine production by CAFs (38).

G-MDSCs promote tumor progression by impeding immune responses, particularly suppression of antigen-specific $\mathrm{CD}^{+} \mathrm{T}$ cells, and promoting tumor invasion and metastasis $(22,34)$. scRNA-Seq highlighted unique G-MDSC subsets with gene signatures associated with enhanced STAT and NF- $\mathrm{KB}$ signaling. A myriad of factors released by the tumor microenvironment activate several different signaling pathways in MDSCs related to the STAT family of transcription factors (46). STAT signaling regulates MDSC expansion, promotes MDSC survival, and promotes increased production of reactive oxygen species by MDSCs. Single-cell transcriptomics of vehicle and anti-CSF1R-treated tumors also identified a unique G-MDSC subset, ApoE G-MDSCs, with abundant expression of Apoe in the vehicle-treated tumors, and marked Apoe downregulation with TAM blockade. The LXR/ ApoE axis has been linked to enhanced MDSC apoptosis and consequent tumor regression (36). Interestingly, this subset was 
A

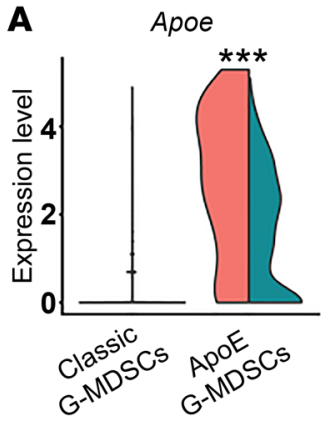

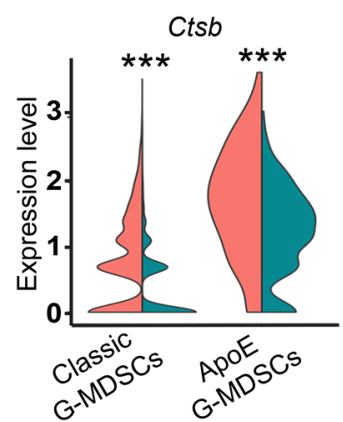

Tumor

B Apoptotic G-MDSCs
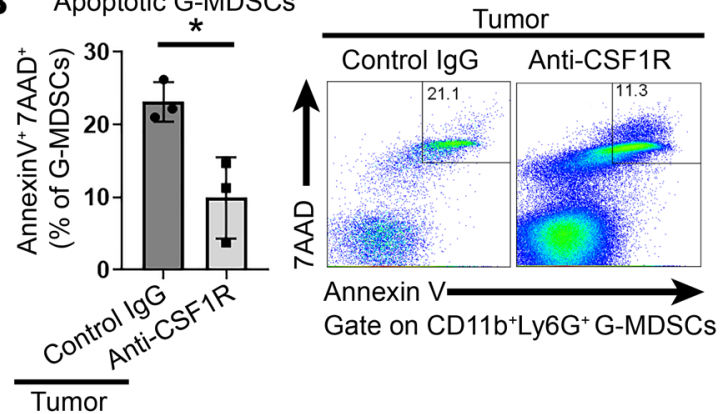
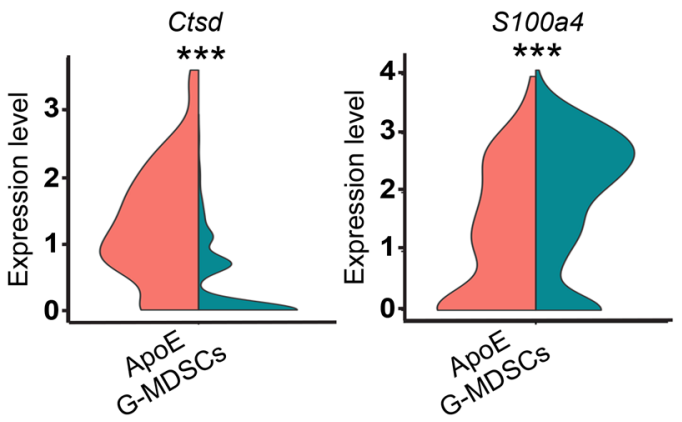

Control IgG

Anti-CSF1R
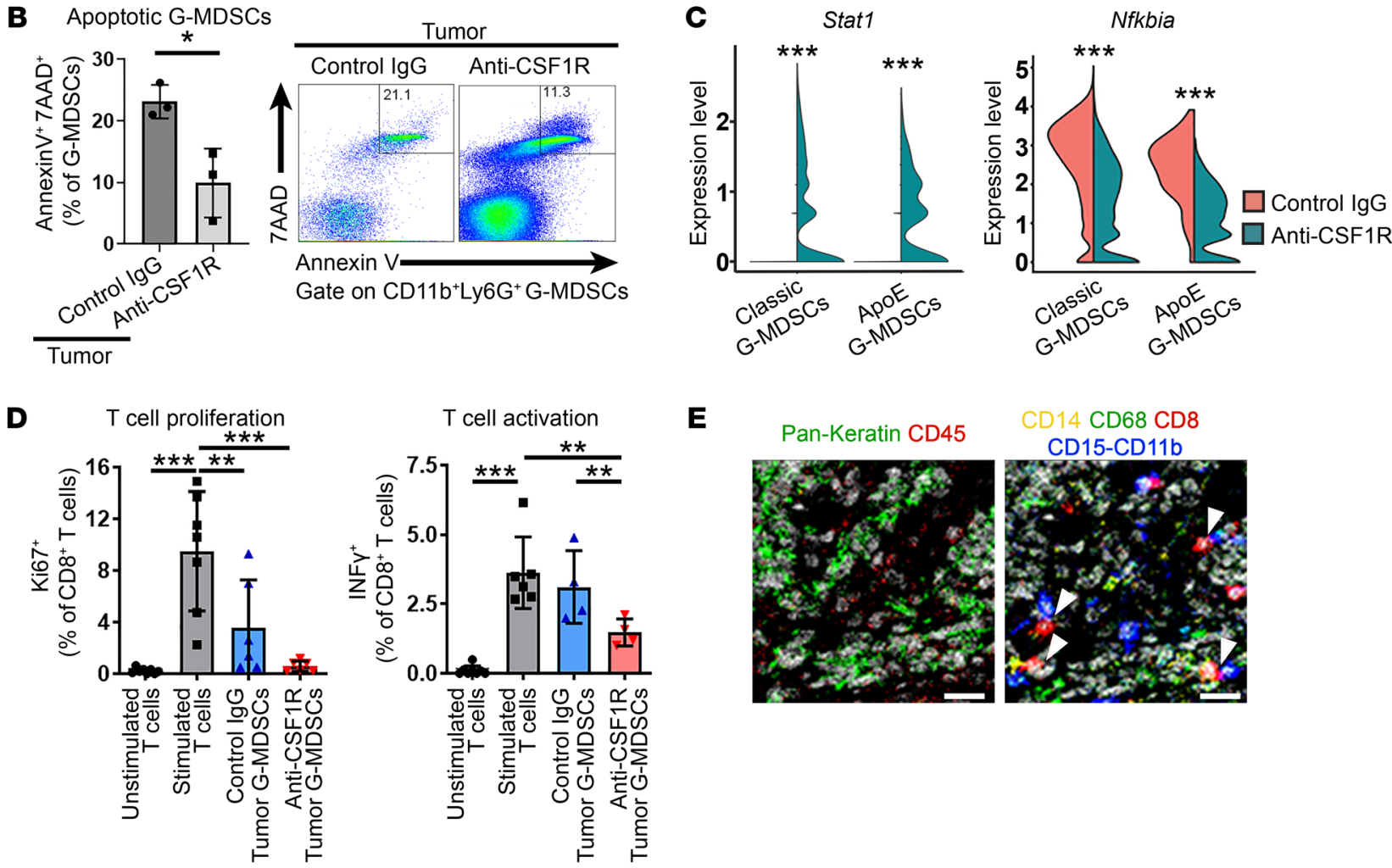

E
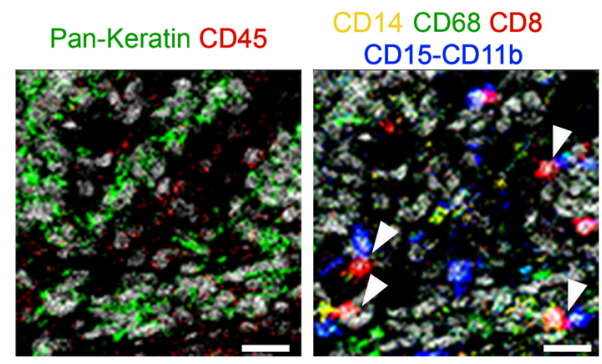

$\mathbf{F}$

Human CCA

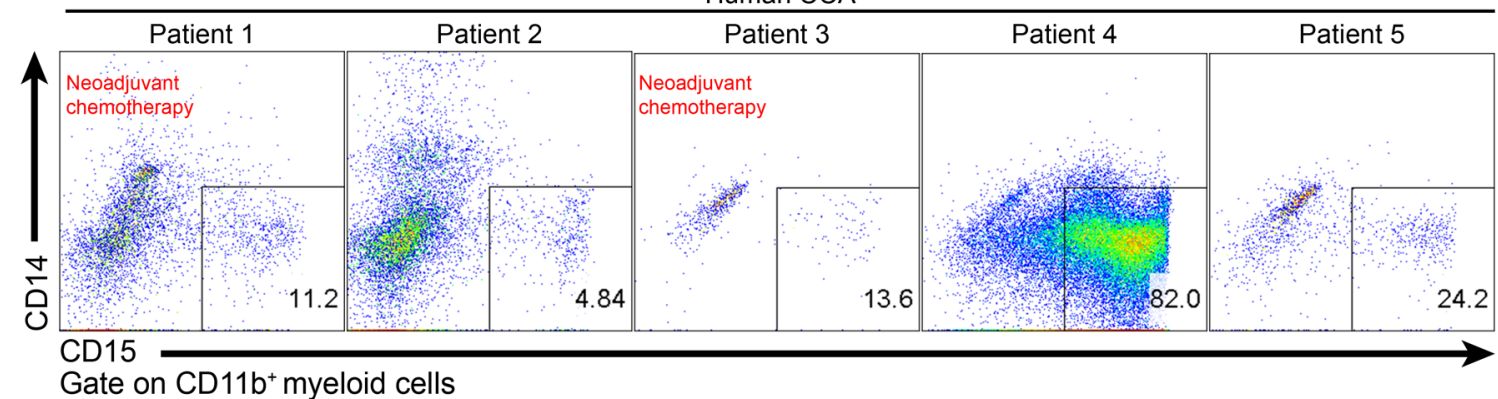

Figure 7. TAM blockade facilitates accumulation of G-MDSC subsets with survival and immunosuppressive properties. (A-D) Tumor growth of 28 days after orthotopic implantation of $1 \times 10^{6} \mathrm{SB}$ (murine CCA) cells in WT mice. Mice were treated from day 14 to day 28 after implantation with control lgC isotype or antiCSF1R (AFS98). (A) Violin plots of expression levels for differentially expressed genes (Apoe, Ctsb, Ctsd, and S100a4) compared between control and anti-CSF1Rtreated tumors. Colors indicate control and anti-CSF1R-treated samples. $P$ values indicate significance of expression differences between control and treatment. (B) Percentage of Annexin $\mathrm{V}^{+} 7 A A D^{+}$G-MDSCs in control or anti-CSF1R-treated tumors $(n \geq 3)$. Representative flow plots show expression of Annexin $V$ and 7AAD in G-MDSCs. (C) Violin plot of expression levels for differentially expressed genes (Stat1 and Nfkbia) compared between control and anti-CSF1R-treated tumors. $P$ values indicate significance of expression differences between control and treatment. (D) Percentage of Ki67 ${ }^{+}$cells of CD8 ${ }^{+}$T cells (CD3 ${ }^{+}$CD8 $8^{+}$(left panel) and percentage of INF- $\gamma^{+}$cells of $C D 8^{+}$T cells (CD3+CD8 $)$(right panel) after 48 hours of coculture with G-MDSCs. (E) Hyperion multiplexed images show several immune cell markers using formalin-fixed, paraffin-embedded tissues from human CCA. Pseudo-colored raw ion images representing the markers of immune cells detected in the region of interest. Left panel shows pan-keratin (green), a CCA marker, and CD45 (red), a leukocyte marker. Right panel shows CD14 (yellow), a monocyte marker; CD68 (green), a macrophage marker; CD8 (red), a CTL marker; CD11b-CD15 (blue), G-MDSC markers. White arrowheads indicate CD8 ${ }^{+}$T cell (red) and G-MDSC (blue) interaction. Scale bars: $10 \mu \mathrm{m}$. (F) Flow plots show expression of CD15-CD14+ G-MDSCs in human CCA. Data are represented as mean \pm SD. Unpaired Student's $t$ test (A-C) and 1-way ANOVA with Bonferroni's post hoc test (D) were used. ${ }^{*} P<0.05$; ${ }^{* *} P<0.01$; ${ }^{* *} P<0.001$. 
strikingly enriched in the anti-CSF1R-treated tumors, suggesting that it may have enhanced survival capabilities via Apoe downregulation. Indeed, we observed a notable reduction in G-MDSC apoptosis in the anti-CSF1R-treated tumors compared with vehicle-treated tumors. Moreover, ApoE G-MDSCs are relevant to the human disease, as we identified a similar subset in a human CCA scRNA-Seq data set.

MDSCs also have a significant association with poor patient outcomes as well as chemotherapy and immunotherapy resistance $(35,47)$. There is limited information on the role of MDSCs in CCA immunobiology. A single study demonstrated an association with cancer stage and presence of circulating MDSCs in 17 patients with CCA (48). We have now demonstrated that G-MDSCs that accumulate with TAM blockade have potent immunosuppressive properties and mediate immune escape by impairing $\mathrm{T}$ cell activity and proliferation. Interestingly, similarly to TAM blockade, G-MDSC blockade alone did not have a tumor-suppressive effect. Moreover, although MDSC blockade using the LXR/ApoE agonist GW3965 has a tumor-suppressive effect across several tumor types (36), GW3965 monotherapy did not reduce tumor burden in our murine CCA model. These data imply that combined inhibition of MDSCs and TAMs is required for a tumor-suppressive effect in CCA. Overall, our observations suggest that multiple layers of resistance encompassing the innate and adaptive immune response contribute to tumor immune evasion in CCA. Hence, combinatorial immunotherapies targeting both the innate and adaptive immune system are more likely to be efficacious in desmoplastic malignancies such as CCA. Indeed, we observed that combined inhibition of MDSCs and TAMs reduced both populations in the CCA TIME and substantially enhanced the antitumor effect of anti-PD-1 therapy in murine CCA. Blockade of either population alone failed to suppress tumor growth. Our observations support the investigation of combination therapies targeting TAMs and MDSCs in human CCA. The toxicities of these combinatorial immune-directed therapies in humans are unclear. Targeting the CSF1/CSF1R axis has been associated with toxicity that limits dose escalation, likely related to systemic depletion of macrophages (49). An alternative therapeutic approach for TAM targeting is employing therapies that reprogram macrophages from a protumor to a tumoricidal phenotype, as this approach circumvents the toxicity associated with total body depletion of macrophages. Various strategies targeting TAM reprogramming are currently under investigation in preclinical and clinical studies (49). Human clinical trial data on therapeutic targeting of MDSCs are limited. In a phase I clinical trial of RGX-104, an LXR/ApoE agonist, significant decreases in MDSC levels in patients were noted (36). Notably, this agent was well tolerated without any dose-limiting toxicities. Therapeutic strategies combining TAM inhibition with MDSC inhibition need to be carefully assessed in an early phase clinical trial.

In summary, we have characterized immunosuppressive myeloid cell populations that foster CCA growth and progression. Blockade of TAMs promotes a compensatory accumulation of G-MDSCs, including a unique ApoE G-MDSC subset that is also present in human CCA. Combining TAM and G-MDSC inhibitors reduced both populations in the tumor site. Moreover, it dramatically enhanced the effect of ICB with anti-PD-1 in our preclini- cal model of CCA. Our findings support the role of combination immunotherapeutic strategies coupling ICB with immunotherapies targeting tumor-promoting myeloid cells in CCA.

\section{Methods}

Syngeneic, orthotopic mouse model of CCA. Murine CCA cells (SB) derived from an oncogene-driven murine model of CCA (23) were maintained in culture medium, as previously described (24). This model of murine CCA is a YAP-driven malignancy that has considerable overlap at the messenger RNA level with human intrahepatic CCA (iCCA); employing 2 public databases (The Cancer Genome Atlas-cholangiocarcinoma [TCGA-CHOL] and the Genotype-Tissue Expression [GTEx] Project), we demonstrated a prominent YAP signature (50) in human CCA compared with adjacent liver and normal liver samples (Supplemental Figure 9, A and B). These observations suggest that oncogenic YAP signaling is activated in human CCA and further strengthen the validity of our murine model in regard to human disease. Mice were anesthetized using 1.5\% to 3\% isoflurane. Under deep anesthesia, the abdominal cavity was opened by a $1 \mathrm{~cm}$ incision below the xiphoid process. A sterile cotton-tipped applicator was used to expose the superolateral aspect of the medial lobe of the liver (24). Using a 27 -gauge needle, $20 \mu \mathrm{L}$ of standard media containing $0.5 \times 10^{6} \mathrm{SB}$ cells was injected into the lateral aspect of the medial lobe. A cotton-tipped applicator was held over the injection site to prevent cell leakage and blood loss. Subsequently, the abdominal wall and skin were closed in separate layers with absorbable chromic 3-0 gut suture material. Four weeks following SB cell implantation, mice were sacrificed and tumor, adjacent liver, and spleen were collected.

Treatments in mice. All treatments were initiated 14 days after SB cell implantation. For the experiments employing anti-CSF1R, mice were randomly assigned to control isotype antibody rat IgG2b, K (Bio $\mathrm{X}$ Cell) or anti-CSF1R (AFS98, Bio X Cell) every 3 days at a dose of $200 \mu \mathrm{g}$ delivered via i.p. injection (13). For the combinatorial treatment experiments, mice were randomly assigned to either control isotype antibody (rat IgG2b, K, Bio X Cell) or vehicle consisting of olive oil (90\%) and DMSO (10\%); anti-CSF1R, $200 \mu$ g every 3 days; anti-Ly6G (1A8, Bio X Cell), $400 \mu$ g i.p. loading dose followed by $200 \mu \mathrm{g} 3$ times a week; anti-PD-1 (G4, Antibody Hybridoma Core, Mayo Clinic), 200 $\mu$ i.p. every other day; or GW3965, 50mg/kg/day diluted in $90 \%$ olive oil and $10 \%$ DMSO by gavage. For the survival study, treatments were administered until animals reached approved conditional score thresholds, as established by the Mayo Clinic IACUC.

Isolation of human tumor-infiltrating immune cells and flow analysis. Human CCA tumor samples were collected during surgical resection and placed in RPMI 1640 media supplemented with penicillin/ streptomycin. Samples were immediately transferred for dissociation with minimal ischemic time. Following removal of fat and fibrous and necrotic areas, fresh tumor tissue specimens were cut into smaller pieces $(<3 \mathrm{~mm})$. Minced sample pieces were transferred to a gentleMACS C Tube (Miltenyi Biotec, catalog 130-093-237) containing 5 $\mathrm{mL}$ digestion enzyme mix prepared according to the manufacturer's instructions (Human Tumor Dissociation Kit, Miltenyi Biotech, catalog 130-095-929). Samples were subsequently dissociated with gentleMACS Octo Dissociator (Miltenyi Biotech) according to the manufacturer's protocol. Following mechanical dissociation, samples were filtered using a $70 \mu \mathrm{m}$ nylon mesh cell strainer (Fisher, catalog 22363548). CD $45^{+}$cells were isolated by CD 45 (TIL) human microbe- 
A

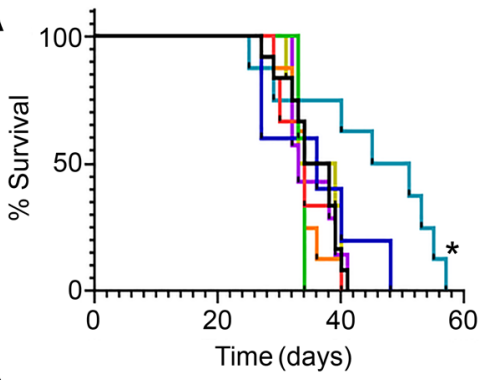

- Control IgG

- Anti-PD-1

Anti-CSF1R

- Anti-Ly6G

- Anti-CSF1R/Anti-Ly6G

- Anti-PD-1/Anti-Ly6G

- Anti-PD-1/Anti-CSF1R

- Anti-PD-1/Anti-CSF1R/Anti-Ly6G

B

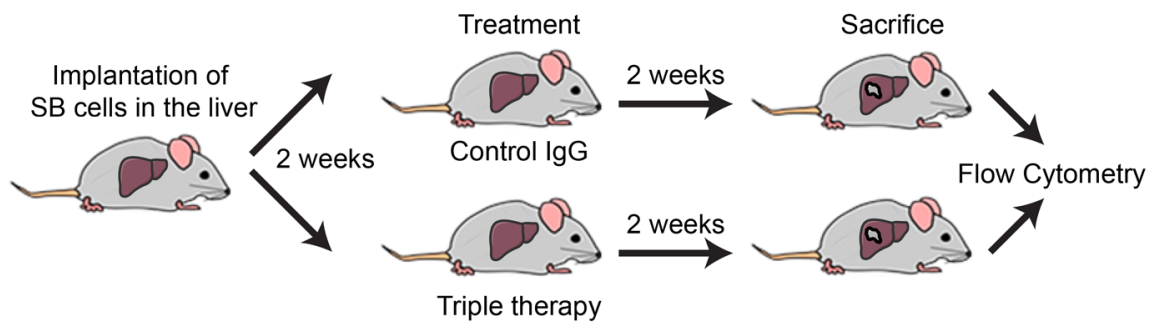

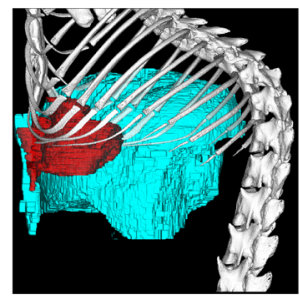

Control Ig

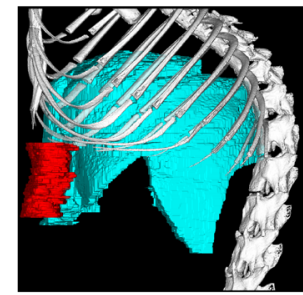

Anti-PD-1/Anti-CSF1R/ Anti-Ly6G
D

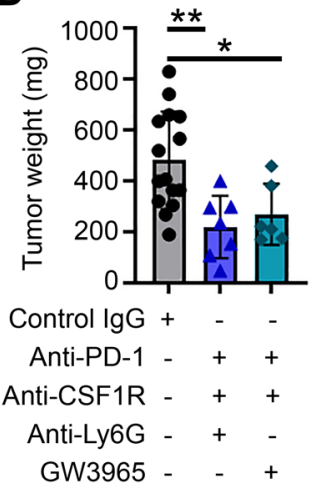

E PD-L1+TAMs

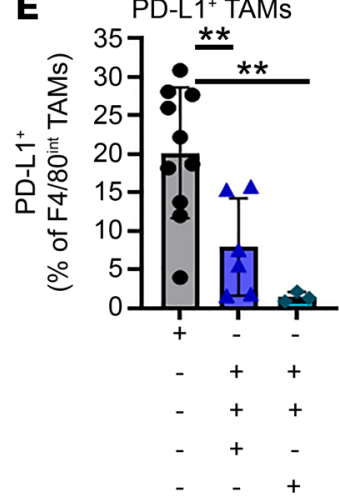

$\mathbf{F}$

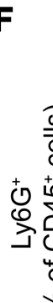

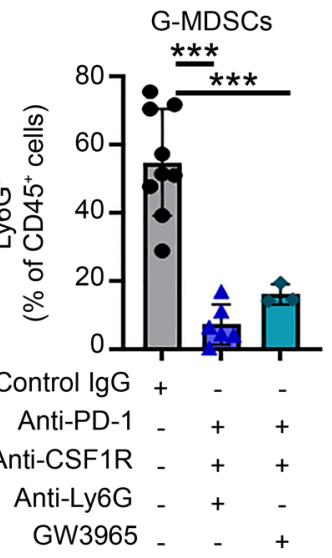

G

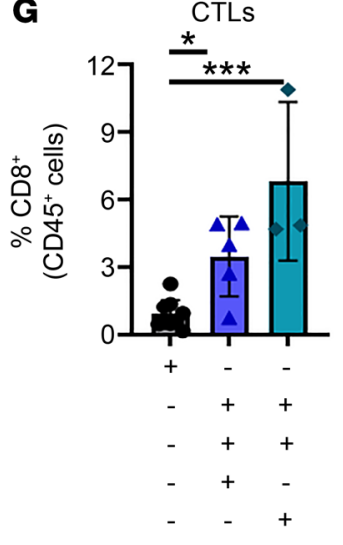

PD-1

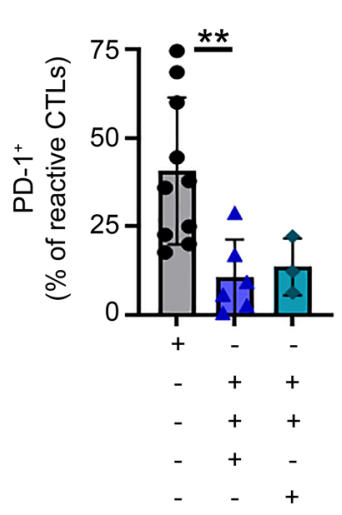

I

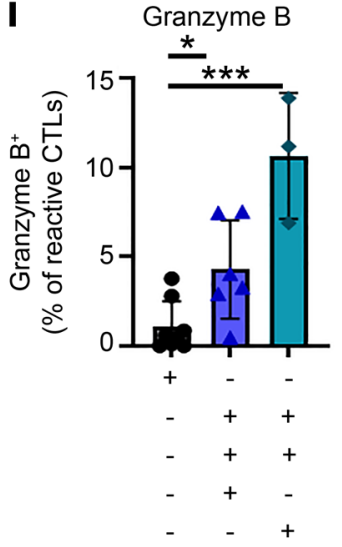

Figure 8. Dual inhibition of G-MDSCs and TAMs potentiates anti-PD-1 therapy. (A-I) Tumor growth of 28 days after orthotopic implantation of $1 \times 10^{6}$ SB (murine CCA) cells in WT mice. Mice were treated from day 14 to day 28 after implantation. (A) Survival curves in mice treated with control rat IgG isotype, anti-PD-1 (G4), anti-CSF1R (AFS98), anti-Ly6G (1A8), GW3965 alone, or in the depicted combinations ( $n \geq 5$ ). (B) Schematic of mouse immunotherapy treatment and characterization. (C) Representative CT image of liver tumor from a contrast reagent-injected mouse treated with control IgC isotype or anti-PD-1+anti-CSF1R+anti-Ly6C 28 days after implantation. The liver is depicted in blue and the tumor in red. (D) Average tumor weights in mg of WT mice treated with control IgG isotype, anti-PD-1+anti-CSF1R+anti-Ly6G, or anti-PD-1+anti-CSF1R+CW3965 ( $n \geq 6$ ) (E) Percentage of PD-L1+ TAMs of $\mathrm{F} 4 / 80^{\text {int }}$ TAMs (CD45 $\left.{ }^{+} \mathrm{CD} 11 \mathrm{~b}^{+} \mathrm{F} 4 / 80^{\text {int }}\right)$ in tumors from WT mice treated with control IgC isotype or anti-PD-1+anti-CSF1R+anti-Ly6C or anti-PD-1+antiCSF1R+CW3965 ( $n \geq 3)$. (F) Percentage of CD11 ${ }^{\text {DimF }} 4 / 80^{-}$CD11 b+Ly6C + G-MDSCs of CD45 cells in tumors from WT mice treated with control IgG isotype or anti-PD-1+anti-CSF1R+anti-Ly6G or anti-PD-1+anti-CSF1R+CW3965 $(n \geq 3)$. (C) Percentage of CD8 ${ }^{+}$CTLs of CD45 ${ }^{+}$cells in tumors from WT mice treated with control IgG isotype or anti-PD-1+anti-CSF1R+anti-Ly6C or anti-PD-1+anti-CSF1R+CW3965 ( $n \geq 3)$. (H) Percentage of PD-1 ${ }^{+}$expressed in CD8 ${ }^{+}$ CD11a+ reactive CTLs (CD3 $\left.{ }^{+} \mathrm{CD}^{+}{ }^{+} \mathrm{CD11a} \mathrm{a}^{+}\right)$in tumors from WT mice treated with control IgC isotype or anti-PD-1+anti-CSF1R+anti-Ly6C or anti-PD-1+antiCSF1R+GW3965 ( $n \geq 3)$. (I) Percentage of granzyme B expressed in CD8+CD11a+ reactive CTLs (CD45+CD3+CD8 + CD11a+) in tumors from WT mice treated with control IgG isotype or anti-PD-1+anti-CSF1R+anti-Ly6C or anti-PD-1+anti-CSF1R+CW3965 $(n \geq 3)$. Data are represented as mean \pm SD. The log-rank Mantel-Cox test $(\mathbf{A})$ and ANOVA with Bonferroni's post hoc test $(\mathbf{C}-\mathbf{H})$ were used. ${ }^{*} P<0.05 ;{ }^{*} P<0.01 ;{ }^{* *} P<0.001$. 
ads (Miltenyi Biotech). Cells were incubated with Fixable Viability Stain 510 (BD Horizon) for 15 minutes, followed by human anti-Fc blocking reagent (Miltenyi Biotec) for 10 minutes before surface staining. Cells were stained, followed by data acquisition on a Miltenyi Biotec MACSQuant Analyzer 10 optical bench flow cytometer. All antibodies were used following the manufacturer's recommendation. Analysis was performed using FlowJo (Tree Star). Forward scatter (FSC) and side scatter (SSC) were used to exclude cell debris and doublets. The following antibodies were used for flow cytometry staining: CD11b-APC-Cy7 (clone ICRF44, BioLegend), CD14-PE (clone M5E2, BioLegend), CD15-BV421 (cloneW6D3, Biosciences), CD68-PE-Cy7 (clone Y1/82A, Biosciences), CD206-PerCP/Cyanine5.5 (clone 15-2, BioLegend), and PD-L1-APC (clone 29E.2A3, BioLegend).

BM transplantation. Eight-week-old male C57BL/6 mice, BL6 SJL mice (CD45.1 allele), or C57BL mice (CD45.2 allele) received acidified water and antibiotics 1 week before irradiation. Then mice were irradiated at $6 \mathrm{~Gy}$ twice within a 6-hour interval. One day following irradiation, 1 million $\mathrm{BM}$ cells were transferred from the donor mice to the recipient mice. Donor mice had a different CD45 allele than recipient mice. Mice were maintained under pathogen-free conditions, and acidified water and antibiotics were provided. Six weeks following irradiation, mice underwent SB cell implantation.

$G-M D S C / T$ cell in vitro coculture. G-MDSCs were isolated from WT mouse tumor, and T cells were isolated from spleen single-cell suspension. T cells and G-MDSCs (ratio 4:1) (51) were cocultured in a 96-well plate with round bottom for 48 hours with CD3/CD28 beads (Gibco Dynabeads, Thermo Fisher Scientific). T cell INF- $\gamma$ transporters were inhibited 5 hours before flow staining with $5 \mu \mathrm{g} / \mathrm{mL}$ of brefeldin A (×100) (BioLegend, 420601) and Monensin (BioLegend 420701). T cells were stained with Fixable Viability Stain 510, CD3APC-Vio770, CD4-PerCP-Vio700, CD8-BV421, Ki67-AF700, and INF- $\gamma$-PE and analyzed by flow cytometry.

Cytometry time-of-flight. Cell preparation and data acquisition for mass cytometry experiments were performed by the Immune Monitoring Core, Mayo Clinic, as described previously (52). Briefly, murine tumor isolates were incubated with $0.5 \mu \mathrm{m}$ of cisplatin solution for 5 minutes. Samples were then incubated at room temperature for 45 minutes with an antibody cocktail of the phenotyping panel (Supplemental Table 5) and fixed with 2\% paraformaldehyde solution. After fixation, samples were resuspended in $30 \mathrm{nM}$ intercalation solution and incubated overnight at $4^{\circ} \mathrm{C}$ before resuspension at $0.5 \times 10^{6}$ cells/ $\mathrm{mL}$ in cell acquisition solution (Fluidigm). Data were acquired with the Helios cytometry time-of-flight (CyTOF) system at a rate of 200 to 400 events per second. Cell cleanup and quality control (QC) analysis were performed with FlowJo, version 10.5.3. Cleaned fcs files were analyzed by the R-based tool Cytofkit, version 3.8 (53-55). Clustering and dimensionality reduction to 10,000 events per file was performed using the Rphenograph algorithm that included all markers used. Visualization of clusters was mapped onto a tSNE map. Antibodies for use in mass cytometry (Supplemental Table 5) were either purchased from the manufacturer (Fluidigm) or were purchased from the designated manufacturer and labeled with metal isotopes using Maxpar X8 antibody-labeling kits (Fluidigm). All labeling was performed by the Mayo Clinic Hybridoma Core.

scRNA-Seq data analysis of murine MDSCs. We used 1× Genomics Cellranger Single Cell Software Suite (version 3.1.0) to generate FASTQ files and to perform alignment to the mm10 reference genome, filtering, barcode counting, and unique molecular identifier (UMI) counting. For subsequent analysis, we followed the integrated analysis workflow in the Seurat package based on SCT transformation (version 3.1) (https://satijalab.org/seurat/v3.1/integration.html) (56, 57). Genes that are expressed in fewer than 3 cells as well as cells that expressed fewer than 200 genes and more than $40 \%$ of mitochondria genes were excluded for downstream analysis in each sample. Each data set was normalized using SCT transform and scaled for each gene across all cells. All data sets were integrated, scaled, and clustered on the low-dimensional space. Enriched gene markers in each cluster conserved across 2 conditions were identified with fold change larger than 1.5, adjusted $P$ value smaller than 0.05 in both conditions, and at least $20 \%$ of cells with expression of a particular gene. Differentially expressed genes within each cluster between the 2 conditions were also detected with fold change larger than 1.5 in either direction and adjusted $P$ value smaller than 0.05 . All clustering and statistical analysis were performed in $\mathrm{R}$ (version 3.5.2).

scRNA-Seq data analysis of human CCA data set. To validate the MDSC-specific genes detected in our mouse data, scRNA-Seq data for 10 human iCCA samples (GSE125449) were processed using Seurat (42). After similar QC and integration steps, the tSNE algorithm was applied to identify cell clusters. Then we defined 2 gene sets. The first gene set consisted of a published human MDSC gene signature (43). The second gene set corresponded to the 40 top genes specific to ApoE G-MDSCs (cluster 1). The assumption was that cells enriched in either of the 2 gene sets were more likely to be MDSCs. The enrichment was calculated for each cell based on the AUCell package (version 1.4.1). AUCell applies a rank-based algorithm and calculates the enrichment of a gene set (gene signatures) based on area under the recovery curve (AUC) (44). The analysis was done separately for the 2 gene sets.

Tissue staining and image acquisition for imaging mass cytometry. All tissue staining and slide preparation were performed by the Mayo Clinic Pathology Research Core. Two formalin-fixed, paraffin-embedded tissue sections derived from human resected CCA specimens $(n=3)$ were stained with our full antibody panel (Supplemental Table 6). The metal tagged antibodies were acquired directly from the manufacturer (Fluidigm). Briefly, slides were baked for 60 minutes in a $60^{\circ} \mathrm{C}$ oven and then cooled for 5 minutes before loading onto a Bond RX Autostainer (Leica) for automated slide preparation before staining. Slides were deparaffinized with xylol and rehydrated through a graded alcohol series, subjected to antigen retrieval in Tris-EDTA for 20 minutes at $100^{\circ} \mathrm{C}$, and blocked with Superblock solution (Thermo Fisher Scientific) before a final wash with PBS supplemented with $0.05 \%$ Tween and $1 \%$ BSA (PBS-TB). Slides were manually stained overnight in a humidity chamber at $4^{\circ} \mathrm{C}$ with a cocktail of the antibodies diluted at the dilution factors indicated in Supplemental Table 6. On the following day, slides were washed with PBS-TB and then stained with an iridium nucleic acid intercalator (Fluidigm) to label cell nuclei. Cells were washed with PBS-TB twice before a final wash with water and drying for 20 minutes at room temperature. Data acquisition was performed on a Helios CyTOF connected to a Hyperion Imaging System (Fluidigm). Optical images of slides were acquired before laser ablation using Hyperion software (version 7.0.5189.0). Ablations were performed at a resolution of 1 micron and at a frequency of $200 \mathrm{~Hz}$. Ten total regions of interest were acquired over 2 consecutive days. Performance stability was ensured through daily calibration using a 3-element full-coverage tuning slide embed- 
ded with the 3 metal elements 89Y, 140Ce, and $175 \mathrm{Lu}$ (Fluidigm). All metals were within the mass range of the CyTOF detector. Images from CCA sections were generated using MCD Viewer software (version 1.0.560.2; Fluidigm).

Micro-CT imaging. On day 14 following SB cell implantation, a $0.1 \mathrm{~mL}$ single tail-vein i.v. injection to conscious mice was performed to administer Mvivo Au (13210, MediLumine). Using microCT (SkyScan 1276 Micro-CT System, Bruker) at a resolution of 20 $\mu \mathrm{m}$, images were acquired at $85 \mathrm{kV}, 200 \mu \mathrm{A}$, with a rotation step of $0.3^{\circ}$ and in $180^{\circ}$. Images were acquired on days 14,21 , and 28 . Beam hardening correction (30\%) was applied using a $1 \mathrm{~mm}$ aluminum filter. The total scanning time for each animal was approximately 5 minutes. The mice were placed supine on an animal bed with realtime visual monitoring of the animal position, respiratory rate, and chamber temperature. All micro-CT image data were acquired in free-breathing mice under isoflurane anesthesia (1.5\%-2\%) without respiratory or cardiac gating. The temperature of the animal bed chamber was maintained at $28-30^{\circ} \mathrm{C}$ to prevent hypothermia. $3 \mathrm{D}$ imaging reconstruction was performed by the X-ray Imaging Core at Mayo Clinic.

Cxcl 2 FISH and costaining for protein. Detection of Cxcl2 mRNA and CK-19 or $\alpha$-SMA protein in formalin-fixed, paraffin-embedded mouse liver tissue was performed using an in situ mRNA and protein costaining protocol described previously (58). Briefly, tissues were deparaffinized, rehydrated, and subjected to heat-mediated antigen retrieval in antigen unmasking solution (Vector Labs). Slides were subsequently incubated in prehybridization solution (3\% BSA in $4 \times$ SSX) for 20 minutes at $54^{\circ} \mathrm{C}$. Tissues were then incubated for 1 hour at $54^{\circ} \mathrm{C}$ with a fluorescein-labeled $\mathrm{Cxcl} 2$ probe (QIAGEN) diluted to $25 \mathrm{nM}$ in hybridization buffer (10\% dextran sulfate in $4 \times$ SSC). Slides were washed and subjected to a tyramide signal amplification step (PerkinElmer). Slides were washed again, blocked in 3\% BSA, and incubated overnight at $4^{\circ} \mathrm{C}$ with a primary antibody to CK-19 (Abcam) or $\alpha$-SMA (Abcam). After overnight incubation, slides were washed and incubated with a secondary antibody (Alexa Fluor 594, Thermo Fisher Scientific), washed again, and mounted in ProLong Gold with DAPI (Thermo Fisher Scientific). Slides were analyzed on a Zeiss 710 Confocal Microscope.

Additional information is available in Supplemental Methods.

Statistics. Experimental statistical analyses were performed using GraphPad Prism software. Student's 2-tailed $t$ test, log-rank (Mantel-Cox) test, and 1-way ANOVA (with Bonferroni's post hoc test) were used throughout, as indicated in the text. Data were considered statistically significant at $P<0.05$.

Study approval. All animal experiments were performed in accordance with protocols approved by the Mayo Clinic IACUC. Clinical information was assimilated from patient records from the Mayo Clinic. Informed consent was obtained for each patient on an ongoing research protocol approved by the Mayo Clinic Institutional Review Board (no. 707-03).

\section{Author contributions}

EL designed and performed experiments, analyzed data, and wrote the manuscript. JY, CC, EB, JW, and KDP conducted experiments. JY, Y Liu, JW, KDP, Y Li, DO, CW, RPG, RLS, and HD analyzed data. CW, RLS, and HD provided constructive suggestions. SI designed and supervised the study, analyzed data, and wrote the manuscript. All authors discussed the results and commented on the manuscript.

\section{Acknowledgments}

EL was supported by the Cholangiocarcinoma Foundation. EB was supported by NIH/National Institute of Diabetes and Digestive and Kidney Diseases grant T32DK07198. RLS was supported by a Department of Defense Career Development Grant (W81XWH-18-1-0297). HD was supported by the Richard M. Schulze Family Foundation and the Mayo Clinic Center for Individualized Medicine Biomarker Discovery Program for Immunotherapy (IMPRESS). SI was supported by the NIH/National Cancer Institute (1K08CA236874), an American Gastroenterology Association Research Scholar Award, the Mayo Center for Biomedical Discovery, the Mayo Center for Cell Signaling in Gastroenterology (Pilot \& Feasibility Award P30DK084567), the Mayo Hepatobiliary Cancer SPORE (P50 CA210964) Career Enhancement Program, the Satter Family Liver Cancer Award, and the Mayo Foundation. This publication was made possible through support by core facilities at the Mayo Clinic, including the Immune Monitoring Core, the Medical Genome Facility Gene Analysis Core, the Pathology Research Core, the Antibody Hybridoma Core, and the X-Ray Imaging Resources Core.

Address correspondence to: Sumera Ilyas, Mayo Clinic College of Medicine and Science, 200 First Street SW, Rochester, Minnesota 55905, USA. Phone: 507.538.4877; Email:rizvi.sumera@mayo.edu.
1. Rizvi S, Khan SA, Hallemeier CL, Kelley RK, Gores GJ. Cholangiocarcinoma - evolving concepts and therapeutic strategies. Nat Rev Clin Oncol. 2018;15(2):95-111.

2. Bertuccio P, et al. Global trends in mortality from intrahepatic and extrahepatic cholangiocarcinoma. J Hepatol. 2019;71(1):104-114.

3. Everhart JE, Ruhl CE. Burden of digestive diseases in the United States Part III: Liver, biliary tract, and pancreas. Gastroenterology. 2009;136(4):1134-1144.

4. Valle J, et al. Cisplatin plus gemcitabine versus gemcitabine for biliary tract cancer. $N$ Engl J Med. 2010;362(14):1273-1281.

5. Dong H, Zhu G, Tamada K, Chen L. B7-H1, a third member of the B7 family, co-stimulates
T-cell proliferation and interleukin-10 secretion. Nat Med.1999;5(12):1365-1369.

6. Sharpe AH, Pauken KE. The diverse functions of the PD1 inhibitory pathway. Nat Rev Immunol. 2018;18(3):153-167.

7. Ueno M. Pembrolizumab for advanced biliary adenocarcinoma: results from the multicohort, phase 2 keynote-158 study. Ann Oncol. 2018;29(suppl_8):viii205-viii270.

8. Binnewies M, et al. Understanding the tumor immune microenvironment (TIME) for effective therapy. Nat Med. 2018;24(5):541-550.

9. Zhu Y, et al. Tissue-resident macrophages in pancreatic ductal adenocarcinoma originate from embryonic hematopoiesis and promote tumor progression. Immunity. 2017;47(3):597.
10. Bayne LJ, et al. Tumor-derived granulocyte-macrophage colony-stimulating factor regulates myeloid inflammation and $\mathrm{T}$ cell immunity in pancreatic cancer. Cancer Cell. 2012;21(6):822-835.

11. Condeelis J, Pollard JW. Macrophages: obligate partners for tumor cell migration, invasion, and metastasis. Cell. 2006;124(2):263-266.

12. Goswami S, et al. Macrophages promote the invasion of breast carcinoma cells via a colony-stimulating factor-1/epidermal growth factor paracrine loop. Cancer Res. 2005;65(12):5278-5283.

13. MacDonald KP, et al. An antibody against the colony-stimulating factor 1 receptor depletes the resident subset of monocytes and tissue- and tumor-associated macrophages but does not inhibit inflammation. Blood. 2010;116(19):3955-3963. 
14. Manthey CL, et al. JNJ-28312141, a novel orally active colony-stimulating factor-1 receptor/ FMS-related receptor tyrosine kinase-3 receptor tyrosine kinase inhibitor with potential utility in solid tumors, bone metastases, and acute myeloid leukemia. Mol Cancer Ther. 2009;8(11):3151-3161.

15. Pyonteck SM, et al. CSF-1R inhibition alters macrophage polarization and blocks glioma progression. Nat Med. 2013;19(10):1264-1272.

16. Veglia F, Perego M, Gabrilovich D. Myeloid-derived suppressor cells coming of age. Nat Immunol. 2018;19(2):108-119.

17. Noy R, Pollard JW. Tumor-associated macrophages: from mechanisms to therapy. Immunity. 2014;41(1):49-61.

18. Dong H, et al. Tumor-associated B7-H1 promotes T-cell apoptosis: a potential mechanism of immune evasion. Nat Med. 2002;8(8):793-800.

19. Lau J, et al. Tumour and host cell PD-L1 is required to mediate suppression of anti-tumour immunity in mice. Nat Commun. 2017;8:14572.

20. Lin $\mathrm{H}$, et al. Host expression of PD-L1 determines efficacy of PD-L1 pathway blockade-mediated tumor regression. JClin Invest. 2018;128(2):805-815.

21. Tang H, et al. PD-L1 on host cells is essential for PD-L1 blockade-mediated tumor regression. J Clin Invest. 2018;128(2):580-588.

22. Gabrilovich DI, Ostrand-Rosenberg S, Bronte V. Coordinated regulation of myeloid cells by tumours. Nat Rev Immunol. 2012;12(4):253-268.

23. Yamada D, et al. IL-33 facilitates oncogene-induced cholangiocarcinoma in mice by an interleukin-6-sensitive mechanism. Hepatology. 2015;61(5):1627-1642.

24. Rizvi S, et al. YAP-associated chromosomal instability and cholangiocarcinoma in mice. Oncotarget. 2018;9(5):5892-5905.

25. Mantovani A, Marchesi F, Malesci A, Laghi L, Allavena P. Tumour-associated macrophages as treatment targets in oncology. Nat Rev Clin Oncol. 2017;14(7):399-416.

26. Liu X, et al. Endogenous tumor-reactive CD ${ }^{+} \mathrm{T}$ cells are differentiated effector cells expressing high levels of CD11a and PD-1 but are unable to control tumor growth. Oncoimmunology. 2013;2(6):e23972.

27. Haderk F, et al. Tumor-derived exosomes modulate PD-L1 expression in monocytes. Sci Immunol. 2017;2(13):eaah5509.

28. Frigola $X$, et al. Identification of a soluble form of B7-H1 that retains immunosuppressive activity and is associated with aggressive renal cell carci- noma. Clin Cancer Res. 2011;17(7):1915-1923.

29. Sideras K, et al. Circulating levels of PD-L1 and Galectin-9 are associated with patient survival in surgically treated hepatocellular carcinoma independent of their intra-tumoral expression levels. Sci Rep. 2019;9(1):10677.

30. Sierro F, et al. A liver capsular network of monocyte-derived macrophages restricts hepatic dissemination of intraperitoneal bacteria by neutrophil recruitment. Immunity. 2017;47(2):374-388.e6.

31. Wang J, Kubes P. A reservoir of mature cavity macrophages that can rapidly invade visceral organs to affect tissue repair. Cell. 2016;165(3):668-678.

32. Murray PJ. Macrophage polarization. Annu Rev Physiol. 2017;79:541-566.

33. Boring $\mathrm{L}$, et al. Impaired monocyte migration and reduced type 1 (Th1) cytokine responses in C-C chemokine receptor 2 knockout mice. JClin Invest. 1997;100(10):2552-2561.

34. Gabrilovich DI. Myeloid-derived suppressor cells. Cancer Immunol Res. 2017;5(1):3-8.

35. Highfill SL, et al. Disruption of CXCR2-mediated MDSC tumor trafficking enhances anti-PD1 efficacy. Sci Transl Med. 2014;6(237):237ra67.

36. Tavazoie MF, et al. LXR/ApoE activation restricts innate immune suppression in cancer. Cell. 2018;172(4):825-840.e18.

37. Zhang H, et al. CXCL2/MIF-CXCR2 signaling promotes the recruitment of myeloid-derived suppressor cells and is correlated with prognosis in bladder cancer. Oncogene. 2017;36(15):2095-2104

38. Kumar V, et al. Cancer-associated fibroblasts neutralize the anti-tumor effect of CSF1 receptor blockade by inducing PMN-MDSC infiltration of tumors. Cancer Cell. 2017;32(5):654-668.e5.

39. Alshetaiwi H, et al. Defining the emergence of myeloid-derived suppressor cells in breast cancer using single-cell transcriptomics. Sci Immunol. 2020;5(44):eaay6017.

40. Pencheva N, Buss CG, Posada J, Merghoub T, Tavazoie SF. Broad-spectrum therapeutic suppression of metastatic melanoma through nuclear hormone receptor activation. Cell. 2014;156(5):986-1001.

41. Li Q, et al. S100A4 protects myeloid-derived suppressor cells from intrinsic apoptosis via TLR4ERK1/2 signaling. Front Immunol. 2018;9:388.

42. Ma L, et al. Tumor cell biodiversity drives microenvironmental reprogramming in liver cancer. Cancer Cell. 2019;36(4):418-430.e6.

43. Murakami S, et al. Yes-associated protein mediates immune reprogramming in pancreatic ductal adenocarcinoma. Oncogene. 2017;36(9):1232-1244.
44. Aibar S, et al. SCENIC: single-cell regulatory network inference and clustering. Nat Methods. 2017;14(11):1083-1086.

45. Liu F, et al. Ceramide activates lysosomal cathepsin $B$ and cathepsin D to attenuate autophagy and induces ER stress to suppress myeloid-derived suppressor cells. Oncotarget. 2016;7(51):83907-83925.

46. Gabrilovich DI, Nagaraj S. Myeloid-derived suppressor cells as regulators of the immune system. Nat Rev Immunol. 2009;9(3):162-174.

47. Zhang S, Ma X, Zhu C, Liu L, Wang G, Yuan X. The role of myeloid-derived suppressor cells in patients with solid tumors: a meta-analysis. PLoS ONE. 2016;11(10):e0164514.

48. Xu XD, et al. Circulating myeloid-derived suppressor cells in patients with pancreatic cancer. HBPD INT. 2016;15(1):99-105.

49. Cassetta L, Pollard JW. Targeting macrophages: therapeutic approaches in cancer. Nat Rev Drug Discov. 2018;17(12):887-904.

50. Yuan WC, et al. NUAK2 is a critical YAP target in liver cancer. Nat Commun. 2018;9(1):4834.

51. Noman MZ, et al. PD-L1 is a novel direct target of HIF-1 $\alpha$, and its blockade under hypoxia enhanced MDSC-mediated T cell activation. JExp Med. 2014;211(5):781-790.

52. Guo Q, et al. Integrin $\beta_{1}$-enriched extracellular vesicles mediate monocyte adhesion and promote liver inflammation in murine NASH. J Hepatol. 2019;71(6):1193-1205.

53. Becher B, et al. High-dimensional analysis of the murine myeloid cell system. Nat Immunol. 2014;15(12):1181-1189.

54. Chen H, Lau MC, Wong MT, Newell EW, Poidinger M, Chen J. Cytofkit: a bioconductor package for an integrated mass cytometry data analysis pipeline. PLoS Comput Biol. 2016;12(9):e1005112.

55. Wong MT, et al. Mapping the Diversity of Follicular Helper T Cells in Human Blood and Tonsils Using High-Dimensional Mass Cytometry Analysis. Cell Rep. 2015;11(11):1822-1833.

56. Butler A, Hoffman P, Smibert P, Papalexi E, Satija R. Integrating single-cell transcriptomic data across different conditions, technologies, and species. Nat Biotechnol. 2018;36(5):411-420.

57. Stuart T, et al. Comprehensive integration of single-cell data. Cell. 2019;177(7):1888-1902.e21.

58. de Planell-Saguer M, Rodicio MC, Mourelatos Z. Rapid in situ codetection of noncoding RNAs and proteins in cells and formalin-fixed paraffin-embedded tissue sections without protease treatment. Nat Protoc. 2010;5(6):1061-1073. 